\title{
Hypersonic Aeroelastic Stability Boundary Computations using Radial Basis Functions for Mesh Deformation
}

\author{
Nicolas Lamorte* and Peretz P. Friedmann ${ }^{\dagger}$ \\ Department of Aerospace Engineering, The University of Michigan, Ann Arbor, MI, 48109-2140, USA
}

\begin{abstract}
This study presents a framework for aeroelastic stability boundary for hypersonic vehicles using CFD and radial basis functions for mesh deformation. The results are presented for two cases: the aeroelastic stability of a two-dimensional typical cross section and a three-dimensional low aspect ratio wing, representative of a control surface of a hypersonic vehicle. Different models of the air mixture are considered: calorifically perfect gas, and imperfect gas models. Chemistry is also considered to account for dissociation of molecules at high temperature. The effect of turbulence and gas modeling on the flutter boundary are investigated. Turbulence can affect the stability boundary by up to $7 \%$. For the flight conditions considered, real gas effects do not modify significantly the flutter Mach number of both systems considered.
\end{abstract}

\section{Nomenclature}

\begin{tabular}{ll}
{$[\mathbf{A}]$} & Transition matrix in the time domain \\
$A$ & Reaction rate parameter \\
$a$ & Non-dimensional elastic axis position, positive aft from midchord \\
$a_{i}$ & Average regression coefficient \\
$b=c / 2$ & Semi-chord \\
$c$ & Chord \\
$C_{P}=\frac{P-P_{\infty}}{q_{\infty}}$ & Pressure Coefficient \\
$c_{p}$ & Specific heat at constant pressure \\
$c_{v}$ & Specific heat at constant volume \\
$E_{a}$ & Activation energy \\
$e_{a}$ & Air internal energy \\
$e_{k}$ & Excitation \\
$F_{Z}, F_{N}$ & Stability metric \\
$f$ & Quantity of interest \\
$H$ & Altitude \\
$h$ & Plunge degree of freedom \\
$h_{a}$ & Air enthalpy \\
$\mathbf{I}$ & Identity matrix \\
$I_{\alpha}$ & Static moment of inertia of the wing section about elastic axis \\
$K_{\alpha}, K_{h}$ & Spring constants in pitch and plunge respectively \\
$\mathbf{K}$ & Structural stiffness matrix \\
$k$ & Reaction rate coefficient \\
$L$ & Lift \\
$M_{e a}$ & Aerodynamic moment \\
$\mathbf{M}$ & Structural mass matrix \\
$\mathbf{M}_{\mathbf{A}}, \mathbf{C}_{\mathbf{A}}, \mathbf{K}_{\mathbf{A}}$ & Aerodynamic influence mass, damping and stiffness matrices \\
\hline
\end{tabular}

${ }^{*}$ Ph. D. Candidate, Student Member AIAA.

${ }^{\dagger}$ François-Xavier Bagnoud Professor, Fellow AIAA. 


\begin{tabular}{|c|c|}
\hline$M$ & Mach number \\
\hline$m$ & Cross sectional mass of the cross section \\
\hline$n$ & Temperature exponent in rate-coefficient expression \\
\hline$N_{m}$ & Number of modes \\
\hline$P$ & Pressure \\
\hline$p$ & Polynomial function \\
\hline$p_{i}$ & Root of the aeroelastic stability equation \\
\hline$\left(p_{n}\right)$ & Polynomial function basis \\
\hline$Q_{i}$ & Generalized aerodynamic load \\
\hline$q_{i}$ & Generalized degree of freedom \\
\hline$R$ & Perfect gas constant \\
\hline$r_{\alpha}$ & Non dimensional radius of gyration \\
\hline$r=\|\boldsymbol{x}\|$ & Euclidian distance from the origin \\
\hline$S_{\alpha}$ & Static mass moment of the wing section about elastic axis \\
\hline$T$ & Temperature \\
\hline$T_{E}$ & Kinetic energy \\
\hline$U_{E}$ & Elastic energy \\
\hline$U_{\infty}$ & Freestream velocity \\
\hline$v_{n}$ & Velocity in the $z$ direction \\
\hline$w$ & Structural deformation in the $z$ direction \\
\hline $\boldsymbol{X}=\{\mathbf{q} ; \dot{\mathbf{q}}\}$ & State variable \\
\hline $\boldsymbol{x}=(x, y, z)$ & Vector position \\
\hline$x$ & Chordwise coordinate \\
\hline$x_{\alpha}$ & $\begin{array}{l}\text { Non dimensional offset between the elasic axis and } \\
\text { the cross-sectional center of gravity, positive for center of gravity behind the axis }\end{array}$ \\
\hline$y$ & Spanwise coordinate \\
\hline$Z_{s}(x, y)$ & Structural shape \\
\hline$z$ & Coordinate normal to the wing \\
\hline \multicolumn{2}{|l|}{ Greek Symbols } \\
\hline$\alpha_{s}$ & Static angle of attack \\
\hline$\alpha$ & Pitch degree of freedom \\
\hline$[\alpha]$ & RBF coefficients \\
\hline$[\beta]$ & RBF coefficients for the polynomial \\
\hline$\gamma$ & Specific heat ratio \\
\hline$\zeta_{i}$ & Damping coefficient \\
\hline$\Theta$ & Integral of the state transition matrix \\
\hline$\mu_{T}$ & Turbulent viscosity \\
\hline$\mu_{L}$ & Laminar viscosity \\
\hline$\rho$ & Air density \\
\hline$\tau$ & Thickness ratio \\
\hline$\Phi$ & State transition matrix \\
\hline$\phi$ & Radial basis function \\
\hline$\psi_{i}(x, y, z)$ & mode shape deformation in the $z$-direction in the $i^{t h}$ mode \\
\hline$\omega_{\alpha}, \omega_{h}$ & Natural frequency in pitch and plunge respectively \\
\hline$\omega_{i}$ & Natural frequency \\
\hline$\omega_{d i}=\omega_{i} \sqrt{1-\zeta_{i}^{2}}$ & Damped frequency \\
\hline$\partial \Omega$ & Wetted surface \\
\hline \multicolumn{2}{|l|}{ Subscript } \\
\hline$\infty$ & Free stream conditions \\
\hline 0 & Total conditions \\
\hline
\end{tabular}


$S$

$\cdot=\frac{d}{d t}$

Symbols

$\|\boldsymbol{x}\|=\sqrt{\sum_{i} x_{i}^{2}}$

Abreviations

CFD

FEM

IG

PG

$\mathrm{RBF}$

$\mathrm{RG}$

SI

SA
Pertaining to the structure

Differentiation with time
Computational fluid dynamics

Finite element model

Ideal gas

Perfect gas

Radial basis function

Real gas

System identification

Spalart Allmaras turbulence model

\section{Introduction, Objectives and Problem Statement}

$\mathrm{H}$ YPERSONIC flight is an active area of research motivated by interest in unmanned rapid response to threats and reusable launch vehicles for affordable access to space. High speed flows are inherently complex and involve phenomena not present in supersonic conditions; e.g. dissociations, ${ }^{1,2}$ chemically reacting flow, viscous interactions and higher levels of aerodynamic heat flux. There are no suitable high speed, high enthalpy tunnels that would permit testing of scaled models of hypersonic vehicles. Furthermore hypersonic aerothermoelastic scaling laws are not available at high Mach numbers. ${ }^{3}$ Therefore, the development of accurate computational aerothermoelastic simulation capabilities is critical for the design and analysis of hypersonic vehicles.

High fidelity numerical simulations of the complex hypersonic flow environment are computationally expensive, and the state of the art is still under development. ${ }^{4}$ Thus, the role of various factors such as real gas effects, chemically reacting flows and complex viscous interactions are not understood. A detailed survey of aeroelastic and aerothermoelastic studies of hypersonic vehicle was presented in Ref 5 . The influence of real gas effects on aeroelastic and aerothermoelastic stability of hypersonic vehicle components (such as a wing representing control surfaces and skin panels) has not been assessed in the literature.

Computational fluid dynamics (CFD) is the only alternative that allows increased modeling complexity of the unsteady aerodynamic loading and heating. Coupling of a CFD code with a computational structural dynamic (CSD) model is considered in this study. A CFD code provides the aerodynamic pressure distribution at the structural interface, while the structural dynamics are represented using the finite element method. The dynamic stability of the aeroelastic system is dependent on the accuracy of the coupling scheme. ${ }^{6-9}$ Due to the classical Eulerian representation of the CFD approach and the Lagrangian formulation of the CSD, a key component of these simulations is the fluid/structure coupling scheme. For the CFD approach, the arbitrary Lagrangian-Eulerian formulation accommodates for the moving boundaries and grid deformation. The case of monolithic schemes which solve the fluid, structure and dynamic mesh equations simultaneously are not appropriate for hypersonic aeroelasticity and aerothermoelasticity. Yet, the conservation of energy and momentum at the fluid/structure interface is important for accurate prediction of the dynamic stability of the aeroelastic system. Several studies have addressed issues where pressure and displacement are calculated within the assumptions of their respective solvers. ${ }^{6,10,11}$ In this case, energy balance may not be satisfied at non-matching boundaries. ${ }^{6}$ Conservation of energy at the interface can be enforced to transfer pressure or displacement between the structural model and the fluid solver. The coupling scheme depends on the assumptions of both solvers and on the mesh deformation method. In addition, the time accurate integration scheme of the equations of motion influences the accuracy of the prediction of aeroelastic stability boundaries. ${ }^{12,13}$

The deformation of the fluid domain is induced by the deformation of the structure at the wetted interface. Radial basis function (RBF) interpolation can be used to deform the entire fluid domain. The principal difficulty is to preserve mesh quality during deformation. RBF have been successfully used to compute large mesh deformations in several aeroelastic applications; ${ }^{11,13-20}$ however it has not been applied to hypersonic 
problems. Other common methods include automatic remeshing, which is computationally expensive, or spring analogy which was used in previous work in hypersonic aeroelasticity and aerothermoelasticity. ${ }^{5}$ The latter is used as a reference to verify the current development of this framework. The overall objective of this study is to develop an analysis for hypersonic aeroelastic studies using CFD simulations with mesh deformation based on RBF combined with a CSD model. The paper has three specific objectives:

1. Verify the aeroelastic framework.

2. Examine coupling using a mesh deformation scheme employing RBF for hypersonic aeroelasticity.

3. Assess the influence of real gas effects and turbulence modeling on hypersonic aeroelastic stability boundary.

\section{I.A. Aeroelastic Studies}

The aeroelastic framework is applied to two cases: (1) the aeroelastic stability of a two-dimensional typical cross section and (2) the aeroelastic stability of a three-dimensional low aspect ratio wing, representative of a control surface of a hypersonic vehicle. These cases have been studied in Ref. 21; therefore, verification of the use of RBF is possible.

\section{I.A.1. Two Degree of Freedom Typical Section}

The flutter Mach number associated with a double wedge typical section is examined. The typical section, shown in Fig. 1, is characterized by pitch $(\alpha)$ and plunge $(h)$ degrees of freedom. The mode shapes correspond to rigid body motions.

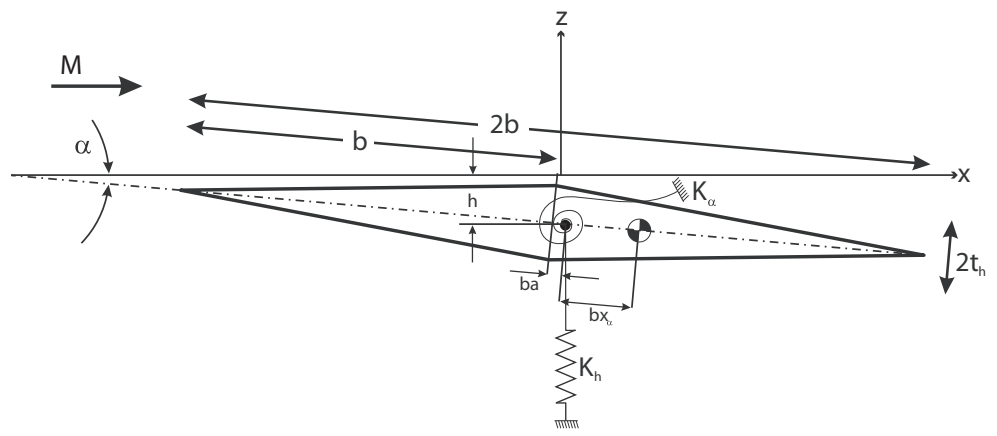

Figure 1. Two degree-of-freedom typical wing section geometry of a supersonic vehicle

The aerodynamic loading is obtained using $3^{\text {rd }}$ order piston theory or CFD calculations. As the freestream Mach number increases, flutter ensues and the flutter Mach number $M_{f}$ corresponds to zero damping in one of the two modes of the system. For this particular problem, the equations of motion (EOM) are given in Eq. (1).

$$
\left[\begin{array}{cc}
m & S_{\alpha} \\
S_{\alpha} & I_{\alpha}
\end{array}\right]\left\{\begin{array}{l}
\ddot{h} \\
\ddot{\alpha}
\end{array}\right\}+\left[\begin{array}{cc}
K_{h} & 0 \\
0 & K_{\alpha}
\end{array}\right]\left\{\begin{array}{l}
h \\
\alpha
\end{array}\right\}=\left\{\begin{array}{c}
-L \\
M_{e a}
\end{array}\right\}
$$

\section{I.A.2. Low Aspect Ratio Wing}

The three-dimensional low-aspect-ratio wing is shown in Fig. 2. The wing is based on the Lockheed F104 Starfighter wing. Its geometry is similar to the fins and control section of prospective long-duration, airbreathing hypersonic vehicles such as the X-43 or NASP. The structural model is extracted from a finite element model created in MSC.NASTRAN and previously studied in Refs. 21 and 22. For aeroelastic stability prediction, the finite element model is reduced to the first five natural modes ${ }^{21}$ which are depicted in Fig. 3. The natural modes are normalized with respect to their modal mass. 


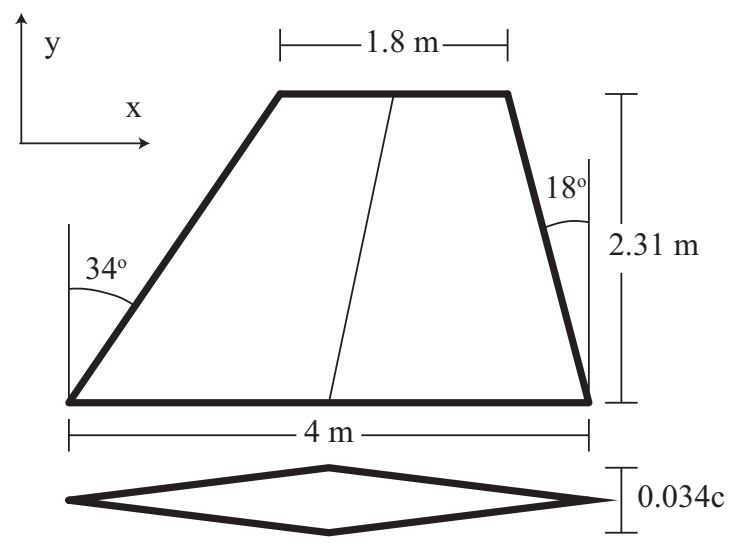

Figure 2. 3D low aspect ratio wing

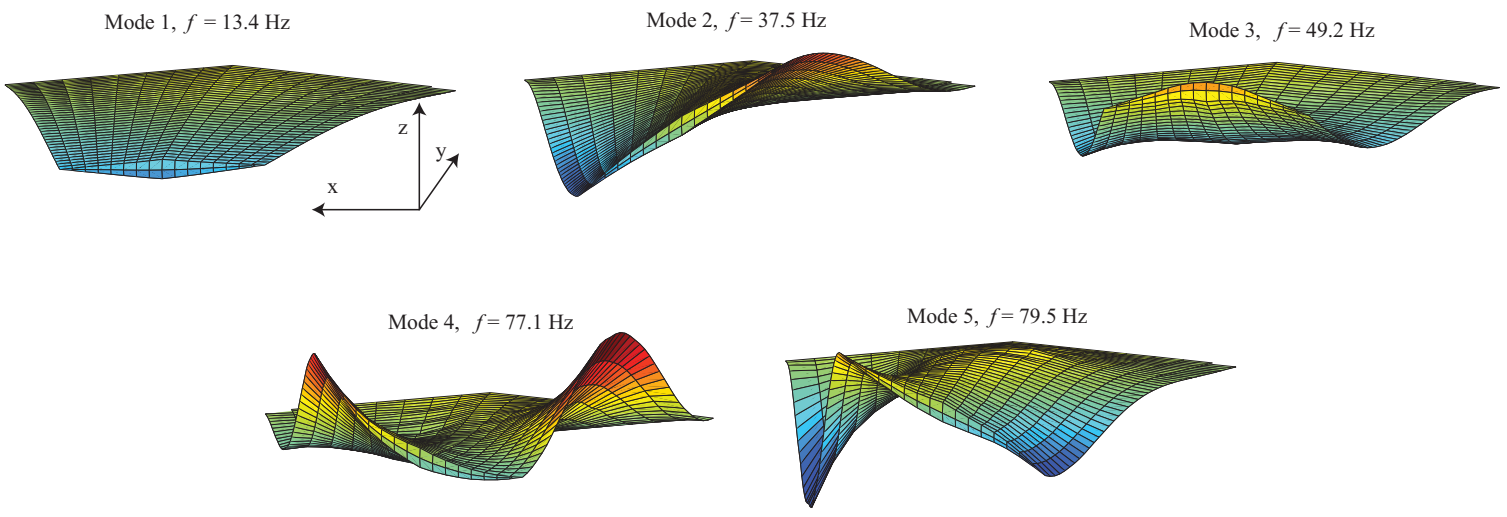

Figure 3. Structural model

\section{Aeroelastic Analysis}

\section{II.A. Equations of Motion and their Solution}

The aeroelastic analysis is illustrated by the flow chart in Fig. 4. The structure is represented by a limited number of mode shapes computed using a finite element model (FEM). The solution of the Navier-Stokes equation is obtained using CFD++, a commercial CFD software capable of accounting for dynamic mesh deformation. The aeroelastic solution is computed by marching the equations of motion in time. At each time step, the deformation of the structure is passed to the mesh deformation capability. The fluid solver computes the pressure distribution for the deformed configuration at the given time step based on the new positions and velocities of the nodes of the fluid mesh. Next, the generalized loads are calculated and the structural deformation is updated. Eventually the aeroelastic transient response is obtained. The aeroelastic system stability is determined from the transient response using two identification methods: the autoregressive moving average approach (ARMA) and the least square curve fit method (LSCFM) used in our earlier studies. ${ }^{21}$ A third approach based on the generalized loads is also considered. Details on each component of the analysis are provided in the following sections. 


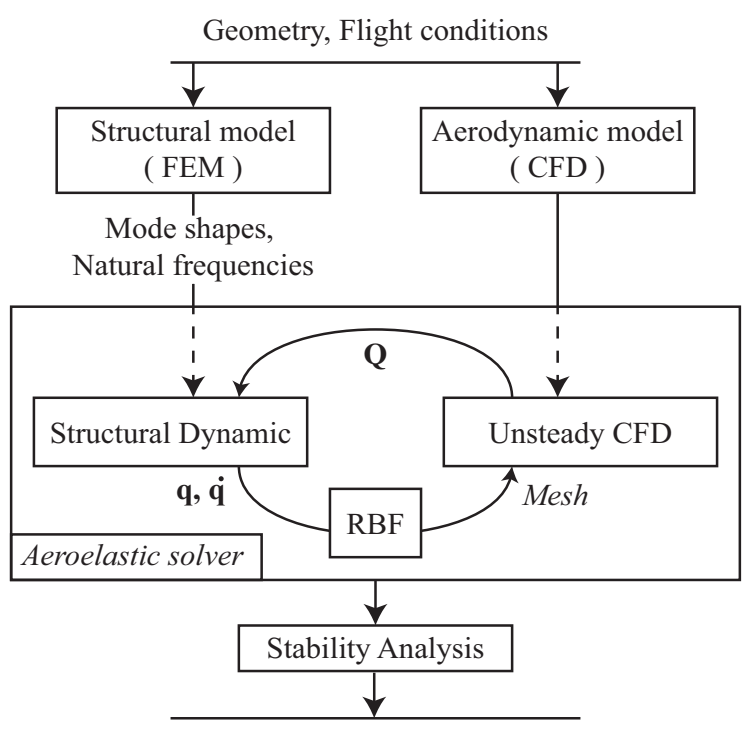

Aeroelastic Boundary

Figure 4. Schematic description of the analysis

\section{II.A.1. Formulation of the Equations of Motion}

Hypersonic vehicle structures are stiff and therefore deformations are small. In this study, the structure is represented by a limited number of its natural modes. This approach is similar to the one employed in the CFL3D code $^{23}$ and Ref. 21. The displacements in the wing mid-plane, $u$ and $v$, are negligible. The out-of-plane displacement, $w(t, \boldsymbol{x})$, at any point of the structure is described by a finite series of modes given by Eq. (2).

$$
w(t, \boldsymbol{x})=\sum_{i=1}^{N_{m}} q_{i}(t) \psi_{i}(\boldsymbol{x})
$$

The equations of motion of the aeroelastic system obtained from Lagrange equations are given in Eq. (3).

$$
\frac{d}{d t}\left(\frac{\partial T_{E}}{\partial \dot{q}_{i}}\right)-\frac{\partial T_{E}}{\partial q_{i}}+\frac{\partial U_{E}}{\partial q_{i}}=Q_{i} \quad i=1, \ldots, N_{m}
$$

From Eq. (3), the final equations of motion (EOM) are obtained as in Eq. (4).

$$
\mathbf{M} \ddot{\mathbf{q}}+\mathbf{K q}=\mathbf{Q}(t, \mathbf{q}, \dot{\mathbf{q}}, \ddot{\mathbf{q}})
$$

The coupling algorithm between the CFD solver and the structural solver used to march in time the EOM is described in the next sections.

\section{II.A.2. Time Integration and Fluid-Structure Coupling}

The fluid and the structure are coupled through the generalized loads. The EOM are given in the state-vector form of Eq. (5).

$$
\left\{\begin{array}{c}
\dot{\mathbf{q}} \\
\ddot{\mathbf{q}}
\end{array}\right\}=\left[\begin{array}{cc}
\mathbf{0} & \mathbf{I} \\
-\mathbf{M}^{-1} \mathbf{K} & \mathbf{0}
\end{array}\right]\left\{\begin{array}{l}
\mathbf{q} \\
\dot{\mathbf{q}}
\end{array}\right\}+\left\{\begin{array}{c}
\mathbf{0} \\
\mathbf{M}^{-1} \mathbf{Q}
\end{array}\right\}
$$

written as:

$$
\{\dot{\boldsymbol{X}}\}=[\mathbf{A}]\{\boldsymbol{X}\}+[\mathbf{B}]\{\boldsymbol{Q}\}
$$


where

$$
[\mathbf{A}]=\left[\begin{array}{cc}
\mathbf{0} & \mathbf{I} \\
-\mathbf{M}^{-1} \mathbf{K} & \mathbf{0}
\end{array}\right] \quad[\mathbf{B}]=\left\{\begin{array}{c}
\mathbf{0} \\
\mathbf{M}^{-1}
\end{array}\right\}
$$

The transition matrix in the time domain, $[\mathbf{A}]$, is constant. The equation of motions can be integrated exactly in time and their analytical solution is given by Eq. (8)

$$
\boldsymbol{X}_{n+1}=e^{[\mathbf{A}] \Delta t} \boldsymbol{X}_{n}+\int_{t_{n}}^{t_{n+1}} e^{[\mathbf{A}]\left(t_{n+1}-\tau\right)}[\mathbf{B}] \boldsymbol{Q}(\tau, \boldsymbol{X}) d \tau
$$

The first term corresponds to the homogeneous response of the structure and the second term accounts for the effect of the aerodynamic loads. The transient response of the aeroelastic system is integrated in time using an explicit method which combines a second order Adams-Bashforth predictor ${ }^{24}$ and a trapezoidal corrector as given in Eqs. (9) and (10), respectively.

$$
\begin{aligned}
& \tilde{\boldsymbol{X}}_{n+1}=\boldsymbol{\Phi} \boldsymbol{X}_{n}+\frac{\boldsymbol{\Theta}}{2}\left(3 \mathbf{Q}\left(\boldsymbol{X}_{n}, t_{n}\right)-\mathbf{Q}\left(\boldsymbol{X}_{n-1}, t_{n-1}\right)\right) \\
& \boldsymbol{X}_{n+1}=\boldsymbol{\Phi} \boldsymbol{X}_{n}+\frac{\boldsymbol{\Theta}}{2}\left(\mathbf{Q}\left(\tilde{\boldsymbol{X}}_{n+1}, t_{n+1}\right)+\mathbf{Q}\left(\boldsymbol{X}_{n}, t_{n}\right)\right)
\end{aligned}
$$

In Eqs. (9) and (10), the state-transition matrix, $\boldsymbol{\Phi}$, and the integral of the state-transition matrix, $\boldsymbol{\Theta}$, are given in Eqs. (11) and (12), respectively.

$$
\begin{gathered}
\mathbf{\Phi}=e^{[\mathbf{A}] \Delta t} \\
\boldsymbol{\Theta}=\int_{t_{n}}^{t_{n+1}} e^{[\mathbf{A}]\left(t_{n+1}-\tau\right)}[\mathbf{B}] d \tau=[\mathbf{A}]^{-1}(\mathbf{\Phi}-\mathbf{I})[\mathbf{B}]
\end{gathered}
$$

The state transition matrix, $\boldsymbol{\Phi}$, is calculated using the Padé approximation with scaling and squaring ${ }^{25}$ implemented in MATLAB's expm function. This method scales the matrix by a power of 2 to reduce the norm to order 1, computes a Padé approximant to the matrix exponential, and then repeatedly squares to undo the effect of the scaling. This process ensures robustness and accuracy of the calculation of the exponential of the matrix.

The time marching method is illustrated in Fig. 5. At the beginning of each time step, the structural deformation at time $t_{n+1}$ is estimated using the generalized loads at the previous and current time step as given by the predictor step, Eq. (9), and represented by the continuous arrows 1 in Fig. 5. Next, the fluid mesh is deformed based on the predicted structure deformation, shown by arrow 2 . The pressure distribution is updated by multiple sub iterations within a time step of the CFD solver to march the Navier Stokes solution to the next time step represented by the arrow 3 . The small lines on it symbolically represent the subiterations of the CFD solver within the time step. Finally, the generalized loads are transferred to the structural solver and the deformation of the structure at time $t_{n}+\Delta t$ is updated using the corrector step given by Eq. (10) based on the generalized loads calculated at time $t_{n+1}$. The corrector step is represented by the dashed arrows 4 .

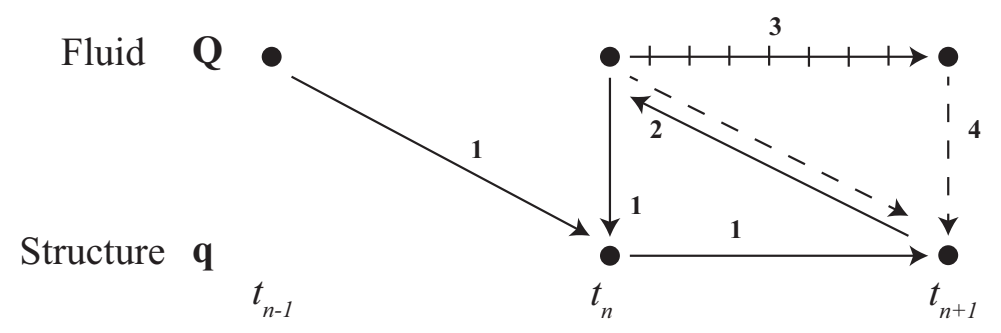

Figure 5. Time marching and coupling approach

The coupling between the fluid and the structure depends on both the mesh deformation method and the calculation of the generalized loads. Both steps are connected to ensure conservation of force and energy through the coupling method. 


\section{II.A.3. Computation of the Generalized Aerodynamic Loads}

The generalized loads are defined by Eq. (13).

$$
Q_{i}(t, \mathbf{q}, \dot{\mathbf{q}}, \ddot{\mathbf{q}})=\int_{\partial \Omega}-\psi_{i}(\boldsymbol{x}) P(\boldsymbol{x}, \mathbf{q}, \dot{\mathbf{q}}, \ddot{\mathbf{q}}) n_{z} d S \quad 1 \leq i \leq N_{m}
$$

The calculation of the generalized loads is performed using the CFD mesh. The integration over the surface of the wing, $\partial \Omega$, is replaced by the summation of the contribution of the faces, $\partial \Omega_{k}$, of the fluid cells that define the wing. The modal shape function, $\psi_{i}$ is evaluated at the centroid of each face, $\boldsymbol{x}_{k}$, to express the generalized loads as given by Eq. (14).

$$
Q_{i} \simeq \sum_{k=1}^{N_{\text {cells }}} \psi_{i}\left(\boldsymbol{x}_{k}\right) \int_{\partial \Omega_{k}}-P(\boldsymbol{x}) n_{z} d S
$$

The quantity $\int_{\partial \Omega_{k}}-P(\boldsymbol{x}) n_{z} d S$ is the contribution of one face to the force in the $z$-direction. It is extracted from the CFD ++ code by the mean of an output file after each time step. The CFD ++ code is not capable of calculating the generalized forces therefore verification of this calculation is infeasible. However, resultant forces and moments can be calculated in a similar manner and compared to the ones calculated by $\mathrm{CFD}++$. The results showed perfect agreement with the present approach. This integration is consistent with the pressure discretization that pertains to the CFD algorithm in CFD ++. Consequently the only approximation made in this approach comes from the calculation of the shape function at the centroid. For consistency, this value is given by the RBF method chosen to deform the mesh, presented next.

\section{II.B. Mesh Deformation Using RBF}

A radial function $(\mathrm{RF}), \phi$, is a scalar function whose value depends only on the distance from the origin, $r=\|\boldsymbol{x}\|$. Radial basis function networks (RBFN) are a well-established tool for multivariate interpolation of both scattered and gridded data. ${ }^{20}$ In aeroelastic applications, the displacement, $w$, is the quantity being interpolated from the fluid-structure interface to the rest of the CFD mesh. Given a set of $N_{s}$ sampling points, also called driving points, $\boldsymbol{x}_{d j}$, at which the deformation is known: $\left(\boldsymbol{x}_{d j}, w_{j}\right)_{j=1, N_{s}}$, the RBF interpolant of $w, \hat{w}$, is constructed in the form given by Eq. (15). The interpolated value at a new point, $\boldsymbol{x}$, depends only on the Euclidian distance between $\boldsymbol{x}$ and the driving points $\left(\boldsymbol{x}_{d j}\right)_{j=1, N_{s}}$.

$$
\hat{w}(\boldsymbol{x})=\sum_{j=1}^{N_{s}} \alpha_{j} \phi\left(\left\|\boldsymbol{x}-\boldsymbol{x}_{j}\right\|\right)+p(\boldsymbol{x})
$$

In Eq. (15), the polynomial $p(\boldsymbol{x})$ and the fitting coefficients $\alpha_{j}$ are determined such that the interpolant, $\hat{w}$, is equal to the actual deformation of $w$ at the sampling points as given in Eq 16 .

$$
w_{i}=\hat{w}\left(\boldsymbol{x}_{d i}\right)=\sum_{j=1}^{N_{s}} \alpha_{j} \phi\left(\left\|\boldsymbol{x}_{d i}-\boldsymbol{x}_{d j}\right\|\right)+p\left(\boldsymbol{x}_{d i}\right) \quad 1 \leq k \leq N_{s}
$$

Introducing $\left(p_{n}(\boldsymbol{x})\right)_{n}$ as a polynomial basis, $p(\boldsymbol{x})$ is written as $\sum_{n=0}^{P} \beta_{n} p_{n}(\boldsymbol{x})$. The coefficients $\beta_{n}$ uniquely determine $p$. To ensure uniqueness of the form given in Eq. (15), i.e. the uniqueness of the coefficients $\alpha_{j}$ and $\beta_{n}$, additional conditions given in Eq. (17) have to be satisfied.

$$
\sum_{j=1}^{N_{s}} \alpha_{j} p_{n}\left(\boldsymbol{x}_{d j}\right)=0 \quad n=0, P
$$

Combining Eqs. (16) and (17), $\alpha_{j}$ and the coefficients $\beta_{n}$ are uniquely defined by solving the linear system written in the form of Eq. (18), where $\left.\boldsymbol{M}_{\boldsymbol{b} \boldsymbol{b}}(\boldsymbol{x})\right)=\left[\phi\left(\left\|\boldsymbol{x}-\boldsymbol{x}_{j}\right\|\right)\right], \boldsymbol{Q}_{\boldsymbol{b}}(\boldsymbol{x})=\left[p_{n}(\boldsymbol{x})\right]_{j=1, N_{s} ; n=0, P}$.

$$
\left[\begin{array}{cc}
\boldsymbol{M}_{\boldsymbol{b b}}\left(\boldsymbol{x}_{\boldsymbol{d}}\right) & \boldsymbol{Q}_{\boldsymbol{b}}\left(\boldsymbol{x}_{\boldsymbol{d}}\right) \\
\boldsymbol{Q}_{\boldsymbol{b}}\left(\boldsymbol{x}_{\boldsymbol{d}}\right)^{T} & \mathbf{0}
\end{array}\right]\left\{\begin{array}{l}
\boldsymbol{\alpha} \\
\boldsymbol{\beta}
\end{array}\right\}=\left\{\begin{array}{c}
w^{S}\left(\boldsymbol{x}_{\boldsymbol{d}}\right) \\
\mathbf{0}
\end{array}\right\}
$$


The displacement at any other point is given by Eq. (19).

$$
w^{F}(\boldsymbol{x})=\left\lfloor\begin{array}{ll}
M_{\boldsymbol{b} \boldsymbol{b}}(\boldsymbol{x}) & \boldsymbol{Q}_{\boldsymbol{b}}(\boldsymbol{x})
\end{array}\right\rfloor\left\{\begin{array}{l}
\boldsymbol{\alpha} \\
\boldsymbol{\beta}
\end{array}\right\}
$$

The computational cost of the mesh deformation can be reduced when assuming small deformations and using a modal representation of the structure. Combining the RBF interpolant in Eqs. (18) and (19) with Eq. (2) yields Eq. (20).

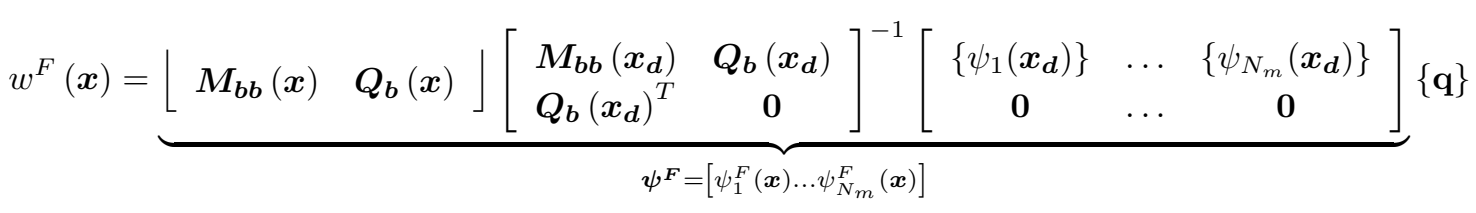

In Eq. (20), each column of the matrix $\boldsymbol{\psi}^{\boldsymbol{F}}$ corresponds to the deformation of the fluid mesh for a given structural mode shape. It is calculated before the start of the aeroelastic simulation. Then, at each time step, the deformation of the fluid mesh is calculated directly from the modal degree of freedom, $\boldsymbol{q}$, by a simple matrix multiplication as given by Eq. (21). It increases memory requirement but reduces the computational cost of each time step since no matrix inversion is needed.

$$
w^{F}(\boldsymbol{x})=\left[\psi_{1}^{F}(\boldsymbol{x}) \ldots \psi_{N_{m}}^{F}(\boldsymbol{x})\right]\{\mathbf{q}\}
$$

In the RBF approach several components are chosen to obtain the best fit possible: the polynomial order, the choice of RF and the set of driver points. In aeroelastic simulations, a polynomial of order 1 is considered such that rigid body motions are exactly interpolated.

Classical radial basis functions, $\phi$, used in engineering applications ${ }^{20}$ are presented in Table 1 and can be grouped in two categories: global and local. In the cases of volume spline, Duchon's thin plate spline, and Hardy's multiquadratics, the amplitude of the radial function increases with the distance from the origin. Consequently the value of the RBF interpolant at a given location is influenced by all the sampling points even the ones that are located far from the interpolated point, the RF is global. For Hardy's inverse multiquadratics and Gaussian, the radial function decreases monotonically to zero with increasing distance from the origin. Therefore the contribution of a sampling point in the interpolant diminishes as the interpolated point is located further away from this sampling point. In the cases of Euclid's hat, and Wedland functions, the radial function is non-zero only at the vicinity of the origin. A sampling point influences only a limited region of the space. Similarly the value of the RBF at a given point depends only on the sampling points located in its vicinity. In addition, the system which determines the RBF coefficients in Eq. (18) is sparse which limits computational cost. Note that the radial functions can be scaled by replacing $\phi(r)$ by $\phi\left(\frac{r}{r_{0}}\right)$ where $r_{0}$ is a reference distance, to obtain a better conditioning of Eq. (18).

The choice of the driving points is important as well. The deformation of the fluid-structure interface is known at the FEM nodes. Several strategies can be considered to deform the fluid mesh based on this information. The most straight forward approach interpolates the deformation from the FEM nodes to the fluid mesh. In this case the driving points are the FEM nodes on the interface between the structure and the fluid. Because of the global form of the RBF interpolant, the deformed interface defined by the fluid mesh does not coincide perfectly with the structural one for fluid points located between adjacent FEM nodes. The FEM mesh is usually more coarse when compared to the fluid mesh, as illustrated schematically in Fig. 6. A simplified CFD mesh is deformed using the FEM nodes of the interface as driver points. The deformed interface given by the FEM in the thick dotted line differs from the CFD one represented by the thin line with crosses.

An alternative is to complement the set of FEM nodes with additional driving points at which the deformation is calculated based on the FEM model. If necessary, every fluid node of the interface can be used as driving points and a RBF interpolation based on those points is created for the rest of the mesh, the interior points. The latter is the method chosen in this study: the deformation of the interface at the fluid mesh is calculated using a piecewise bilinear interpolation between the FEM nodes.

To quantify the error at the fluid-structure interface due to RBF interpolation, different error metrics are considered and given in Eqs. (22) to (24) where $w_{r e f}$ is either the analytical form of the deformation or 
Table 1. Classical radial basis functions

\begin{tabular}{|c|c|c|}
\hline $\mathrm{RBF}$ & Support & $\phi(r)$ \\
\hline Volume spline & & $r$ \\
\hline Duchon's thin plate spline & & $r^{2} \log (r)$ \\
\hline Hardy's multiquadratics & & $\left(1+r^{2}\right)^{1 / 2}$ \\
\hline Hardy's inverse multiquadratics & & $\left(1+r^{2}\right)^{-1 / 2}$ \\
\hline Euclid's Hat function & compact & $\left\{\begin{array}{cl}\pi\left(\frac{r^{3}}{12}-r+\frac{4}{3}\right) & \text { if } \quad r<2 \\
0 & \text { otherwise }\end{array}\right.$ \\
\hline Wedland $C^{0}$ & compact & $\left\{\begin{array}{cl}(1-r)^{2} & \text { if } \quad r<1 \\
0 & \text { otherwise }\end{array}\right.$ \\
\hline Wedland $C^{2}$ & compact & $\begin{array}{cl}(1-r)^{4}(4 r+1) & \text { if } \quad r<1 \\
0 & \text { otherwise }\end{array}$ \\
\hline Gaussian & & $\exp \left(-r^{2}\right)$ \\
\hline
\end{tabular}

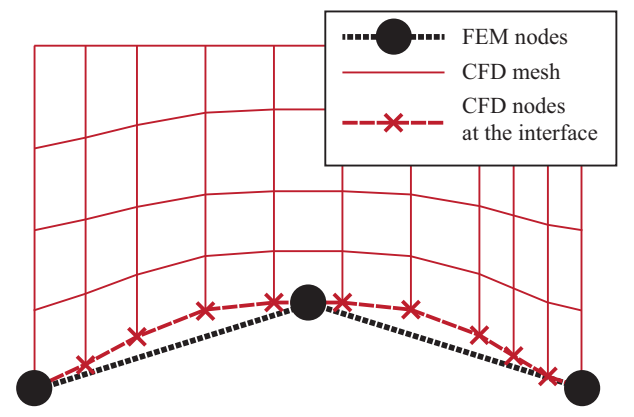

Figure 6. Illustration of the usage of RBF for mesh deformation

a piecewise interpolation between the FEM points.

$$
\begin{aligned}
L_{2} & =\sqrt{\frac{1}{N} \sum \frac{\left(\hat{w}-w_{\text {ref }}\right)^{2}}{\max \left(w_{\text {ref }}^{2}\right)}} \\
L_{1} & =\frac{1}{N} \frac{\sum\left\|\hat{w}-w_{\text {ref }}\right\|}{\max \left(\left\|w_{\text {ref }}\right\|\right)} \\
L_{\text {max }} & =\frac{\max \left(\left\|\hat{w}-w_{\text {ref }}\right\|\right)}{\max \left(\left\|w_{\text {ref }}\right\|\right)}
\end{aligned}
$$

\section{II.C. Perfect Gas and Real Gas Modeling}

Solving the Navier-Stokes equations for hypersonic flow is computationally expensive; however, the representation of the air flow over the structure can account for viscous and high temperature effects. The commercial code CFD++ solves the Navier-Stokes equations using a point-implicit finite volume approach. The one equation Spalart Allmaras turbulence model is considered in this study. Two simple models of the fluid are available perfect (PG) and thermally imperfect or ideal (IG) gas, and presented in Table 2. In addition, chemical reactions can be incorporated to account for the dissociation of molecules at high temperatures, referred to as real gas effects (RG).

In both PG and IG gas models, the fluid is modeled by a single species; there is no chemical reaction. Pressure, temperature, and density are related by the same equation of state. In the perfect gas model, the specific heats are constant. Therefore internal energy, $e_{a}$, and enthalpy, $h_{a}$, are linear functions of 


\begin{tabular}{ccc}
\hline \hline & Perfect Gas (PG) & Ideal Gas (IG) \\
\hline Equation of state & \multicolumn{3}{c}{$P=\rho R T$} \\
\hline Internal energy & $\partial e_{a}=c_{v} \partial T$ & $\partial e_{a}=c_{v}(T) \partial T$ \\
Enthalpy & $\partial h_{a}=c_{P} \partial T$ & $\partial h_{a}=c_{P}(T) \partial T$ \\
$\gamma$ & $\gamma=$ constant & $\gamma(T)$ \\
\hline \hline
\end{tabular}

Table 2. Perfect and Ideal gas models

temperature. The specific heat ratio, $\gamma=\frac{c_{p}}{c_{v}}$ is constant, assumed to be equal to 1.4. Under these assumptions, limitations of the model manifest themselves at high speed. The compression ratio $\frac{\rho_{0}}{\rho_{\infty}}$ is artificially limited to be below 6 and stagnation temperature is unrealistically high. Therefore, this model is often inadequate for hypersonic high altitude flight or reentry problems with high gas temperatures accompanied by non-equilibrium flows. ${ }^{1}$

For ideal gas, specific heats are not constant with temperature, as illustrated in the expression of internal energy and enthalpy in Table 2. Similarly $\gamma$ is a function of temperature. Polynomial fitting using experimental data can estimate the internal energy and enthalpy of air as functions of temperature. In ideal gas, the compression ratio can increase up to 20 and produce a significant reduction in the stagnation temperature when compared to perfect gas prediction.

Details of the real gas model, taken from Ref. 26, are given in Table 3. It contains 5 species and 8 reactions to account for the dissociation of $\mathrm{O}_{2}, \mathrm{~N}_{2}$, and $\mathrm{NO}$. Chemical equilibrium is assumed and there is no ionization. For each reaction, the reaction constant is given by $k=A T^{\eta} \exp \left(-\frac{E_{a}}{R T}\right)$.

Table 3. Reactions

\begin{tabular}{ccccc}
\hline \hline Reactions & $\eta$ & $A\left(\mathrm{~cm}^{3} / \mathrm{mol}\right)$ & $\frac{E_{a}}{R}(\mathrm{~K})$ & \\
\hline$O_{2}+M \rightarrow 2 O+M$ & -1.50 & $2.0 \times 10^{21}$ & 59500 & $M=N_{2}, O_{2}, N O$ \\
$O_{2}+M \rightarrow 2 O+M$ & -1.50 & $1.0 \times 10^{22}$ & 59500 & $M=N, O$ \\
$N_{2}+M \rightarrow 2 N+M$ & -1.60 & $7.0 \times 10^{21}$ & 113200 & $M=N_{2}, O_{2}, N O$ \\
$N_{2}+M \rightarrow 2 N+M$ & -1.60 & $3.0 \times 10^{22}$ & 113200 & $M=N, O$ \\
$N O+M \rightarrow N+O+M$ & 0.00 & $5.0 \times 10^{15}$ & 75500 & $M=N_{2}, O_{2}, N O$ \\
$N O+M \rightarrow N+O+M$ & 0.00 & $1.1 \times 10^{17}$ & 75500 & $M=N, O$ \\
\hline$N_{2}+O \rightarrow N O+N$ & -1.00 & $6.4 \times 10^{17}$ & 38400 & \\
$N O+O \rightarrow O_{2}+N$ & 0.00 & $8.4 \times 10^{12}$ & 19450 & \\
\hline
\end{tabular}

Next a computationally efficient model of the aerodynamic loads is presented.

\section{II.D. Approximate Aerodynamic Loading Using Piston Theory}

Piston theory has been used extensively in numerous studies on hypersonic aeroelasticity. ${ }^{5}$ Third order piston theory (PT) was successfully used in flutter prediction of a typical supersonic control surface in hypersonic flow and it was shown to outperform other approximate approaches when compared against CFD calculation. ${ }^{5}$ Therefore, this computationally efficient model can be used to bracket the flutter Mach number before running expensive CFD simulations. Piston theory gives a point-wise relationship between local deformation and pressure, as given in Eq. (25). It assumes perfect gas, inviscid and isentropic flow.

$$
\frac{P-P_{\infty}}{P_{\infty}}=\gamma\left[\frac{v_{n}}{a_{\infty}}+\frac{\gamma+1}{4}\left(\frac{v_{n}}{a_{\infty}}\right)^{2}+\frac{\gamma+1}{12}\left(\frac{v_{n}}{a_{\infty}}\right)^{3}\right]
$$

The pressure depends on the free stream conditions and $v_{n}$, the velocity of the air at the boundary normal 
to the mean surface of the wing, given in Eq. (26).

$$
v_{n}(x, y, z)=\left\{\begin{array}{cc}
\frac{\partial w}{\partial t}+U_{\infty}\left[\frac{\partial}{\partial x}\left(Z_{s}+w\right)+\alpha_{s}\right] & z>0 \\
-\frac{\partial w}{\partial t}+U_{\infty}\left[\frac{\partial}{\partial x}\left(Z_{s}-w\right)-\alpha_{s}\right] & z<0
\end{array}\right.
$$

The $x$-axis refers to the free stream velocity direction and $Z_{s}$ the normal coordinate of the undeformed geometry at a specific point and $\alpha_{s}$ is the angle of attack. Additional details are out of the scope of this study; however, note that piston theory gives a quasi-steady representation of the aerodynamic loading and that the resulting generalized loads are linear with respect to small deformations.

\section{Stability Boundary Calculation}

The three system identification (SI) methods described in this section assume that the aeroelastic system can be treated as linear dynamic system. It means that a modification of the initial conditions linearly affects the transient response of the system. This assumption permits the identification of the frequencies and damping coefficients of the aeroelastic system and thus predict the onset of instability. In a numerical approach, in order to make sure that all the modes are excited, the initial conditions are set such that all components of the modal velocities, $\dot{q}$, are non-zero.

\section{III.A. ARMA}

The Autoregressive Moving-Average (ARMA) method is an efficient system identification method used to compute the frequencies and corresponding damping coefficients from the transient response of the aeroelastic system. It has been used for flutter prediction of hypersonic systems in Refs. 21 and 27.

The aeroelastic response, $w$ of the structure at a given point is modeled by Eq. (27), where $w_{k}$ is the displacement of a given point of the structure and $e_{k}$ is the excitation at time $k \Delta t$. It assumes that at each time step the response of the system is a linear combination of the response at the $n$ previous time steps and a linear combination of the input $e$ at current and previous time steps $k$ to $k-m$. For this reason, ARMA approach is applicable to systems which behavior can be identified as linear combination of damped harmonic oscillations. ${ }^{21}$

$$
w_{k}+a_{1} w_{k-1}+a_{2} w_{k-2}+\ldots+a_{n} w_{k-n}=b_{1} e_{k-1}+b_{2} e_{k-2}+\ldots+b_{m} e_{k-m}
$$

In flutter studies, $n=2 N_{m}$ and $m=1$ are typical values. The choice of $m=1$ is only required to account for a non-zero static offset. In order to accurately identify the modal response of the aeroelastic system, all modes need to be excited. Based on the aeroelastic transient response, the coefficients $\left(a_{i}\right)$ are calculated. From those coefficients, the frequencies and damping of the system are calculated. The ARMA method only requires the transient response at one point to estimate frequencies and damping.

\section{III.B. Least Squares Curve Fit}

The least squares curve fit method (LSCFM) is a common and robust way of identifying the damping coefficients and frequencies of a signal. The transient response of the structure is assumed to be a linear combination of damped harmonic responses as given in Eq. (28).

$$
\tilde{w}(t)=a_{0}+\sum_{i=1}^{N_{m}} e^{-\zeta_{i} \omega_{i} t}\left[a_{i} \cos \left(\omega_{d i} t\right)+b_{i} \sin \left(\omega_{d i} t\right)\right]
$$

The unknown frequencies and damping are calculated by minimizing the squared error between the aeroelastic transient response and the form in Eq. (28). The resulting minimization problem is given in Eq. (29).

$$
\min _{\omega_{i}, \zeta_{i}, a_{i}, b_{i}}\left(\sum_{k=1, N_{t}}\left[w_{k}-\tilde{w}\left(t_{k}\right)\right]^{2}\right)
$$

In the current implementation, it is solved using two to five iterations of the FMINCON function available in MATLAB. The solution of this highly nonlinear optimization problem is expensive and sensitive to the 
initial guess of the different variables. However error estimates of the approximation are available and convergence of the fit can be assessed.

\section{III.C. p-Method}

The p-method is a well-known approach ${ }^{28,29}$ to determine the stability of an aeroelastic system of which the equations of motion are linear. It requires that the generalized loads are a known function of the generalized degrees of freedom and their time derivatives, as given for instance in Eq. (30).

$$
\mathbf{Q}(t, \mathbf{q}, \dot{\mathbf{q}}, \ddot{\mathbf{q}})=\mathbf{M}_{\mathbf{A}} \ddot{\mathbf{q}}+\mathbf{C}_{\mathbf{A}} \dot{\mathbf{q}}+\mathbf{K}_{\mathbf{A}} \mathbf{q}
$$

To determine the stability of the system, the EOM given in Eq. (4) are combined with Eq. (30), assuming $\mathbf{q}=\mathbf{q}_{0} e^{p t}$. The resulting equation is called the flutter equation given in Eq. (31).

$$
\left\{\left(\mathbf{M}-\mathbf{M}_{\mathbf{A}}\right) p^{2}-\hat{\mathbf{C}}_{\mathbf{A}} p+\mathbf{K}-\hat{\mathbf{K}}_{\mathbf{A}}\right\} \mathbf{q}_{0}=0
$$

Non-zero solutions of the flutter equation are obtained for values of $p$ that satisfy the characteristic equation of the aeroelastic system given in Eq. (32).

$$
\operatorname{det}\left[\left(\mathbf{M}-\mathbf{M}_{\mathbf{A}}\right) p^{2}-\hat{\mathbf{C}}_{\mathbf{A}} p+\mathbf{K}-\hat{\mathbf{K}}_{\mathbf{A}}\right]=0
$$

The frequencies and damping of the aeroelastic system are given by the roots, $p_{i}$, of Eq. (32). In order to be consistent with the ARMA and LSCFM methods, the frequencies and corresponding damping coefficients are uniquely identified by the real and imaginary parts of $p_{i}$ as given by Eq. (33).

$$
p_{i}=-\zeta_{i} \omega_{i}+\boldsymbol{i} \omega_{d i}
$$

In the present study, the aerodynamic loads are computed using unsteady CFD and the analytical form given in Eq.(30) is not available. However, a linear least squares fit can be used to estimate the aerodynamic influence matrices. Based on the form of piston theory and its good agreement with CFD in previous studies, it is assumed that $\mathbf{M}_{\mathbf{A}}$ is negligible. The estimates of the aerodynamic stiffness and damping matrices are given by solving the least squares problem stated in Eq. (34).

$$
\min _{\hat{K}_{A i j}, \hat{C}_{A i j}}\left(\sum_{k=1, N_{t}}\left\|\mathbf{Q}_{\mathbf{k}}-\mathbf{C}_{\mathbf{A}} \dot{\mathbf{q}}_{\mathbf{k}}-\mathbf{K}_{\mathbf{A}} \mathbf{q}_{\mathbf{k}}\right\|^{2}\right)
$$

The solution is obtained using a linear least squares approach ${ }^{30}$ and given in Eq. (35).

$$
\left[\hat{\mathbf{K}}_{\mathbf{A}}, \hat{\mathbf{C}}_{\mathbf{A}}\right]^{T}=\left(\mathbf{X}^{T} \mathbf{X}\right)^{-1} \mathbf{X}^{T} \mathbf{Q} \quad \text { where } \quad \mathbf{X}=\left[\begin{array}{ccc}
\mathbf{q}_{1} & \ldots & \mathbf{q}_{N_{t}} \\
\dot{\mathbf{q}}_{1} & \ldots & \dot{\mathbf{q}}_{N_{t}}
\end{array}\right]^{T} \quad \text { and } \quad \mathbf{Q}=\left[\begin{array}{c}
\mathbf{Q}_{1}^{T} \\
\vdots \\
\mathbf{Q}_{N_{t}}^{T}
\end{array}\right]
$$

This method uses the generalized loads. Both previous methods used only the deformation at one or several points and do not take full advantage of all the information available in a computational framework. For a perfectly linear dynamic system, the frequencies and damping coefficients calculated by each approach are equal.

\section{Results}

In this section the aeroelastic simulations are performed for the typical section and low aspect ratio wing and compared with results obtained in Ref. 21. 


\begin{tabular}{ccc}
\hline \hline Parameter & Value & Units \\
\hline$a$ & 0.1 & - \\
$c$ & 2.35 & $\mathrm{~m}$ \\
$\tau$ & 3.36 & $\%$ \\
$m$ & 94.2 & $\mathrm{~kg} / \mathrm{m}$ \\
$r_{\alpha}$ & 0.484 & $\mathrm{~kg} / \mathrm{m}$ \\
$x_{\alpha}$ & 0.2 & - \\
$\omega_{h}$ & 13.4 & $\mathrm{~Hz}$ \\
$\omega_{\alpha}$ & 37.6 & $\mathrm{~Hz}$ \\
\hline \hline
\end{tabular}

Table 4. Baseline configuration for the typical section

\section{IV.A. Two Dimensional Typical Section}

The stability of the two-dimensional typical section is investigated using CFD. The Navier-Stokes equations are solved with a Spalart-Allmaras turbulence model. Goldberg turbulence model, which is an acceptable turbulence model for hypersonic flow, ${ }^{4}$ is also considered for comparison. The boundary condition at the wall corresponds to an adiabatic wall (AD). The system is characterized by the parameters given in Table 4, which were taken from Ref. 21.

In hypersonic flow the domain of dependency of the pressure on the airfoil is limited. Therefore it is physically acceptable to consider only a limited region around the airfoil. In the fluid mesh depicted in Fig. 7(a), the leading edge shock is contained inside the mesh for all the flight conditions considered. The boundary layer returns to supersonic speeds before it exits the fluid mesh. In addition, the spacing close to the airfoil ensures that the required condition for accurate capture of turbulent flows, $y^{+}<1$, is satisfied. Finally to ensure that the grid is symmetric, the lower half is imported to CFD ++ and replicated in a symmetric manner to form the upper half of the grid.

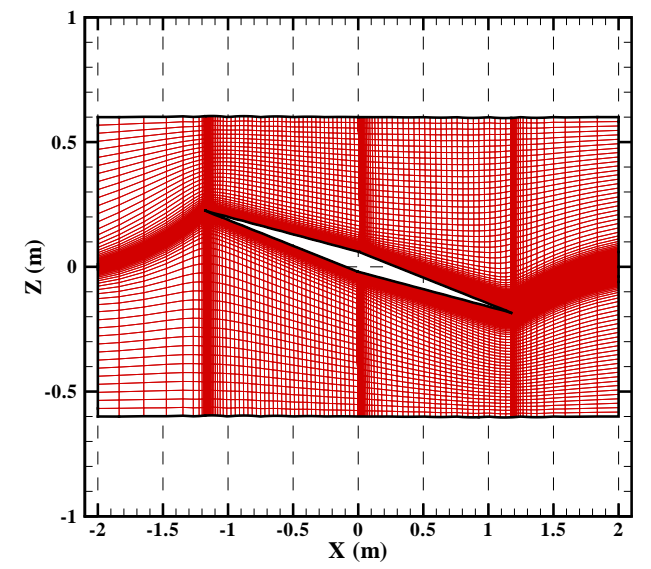

(a) Grid deformation for $\alpha=10^{\circ}$

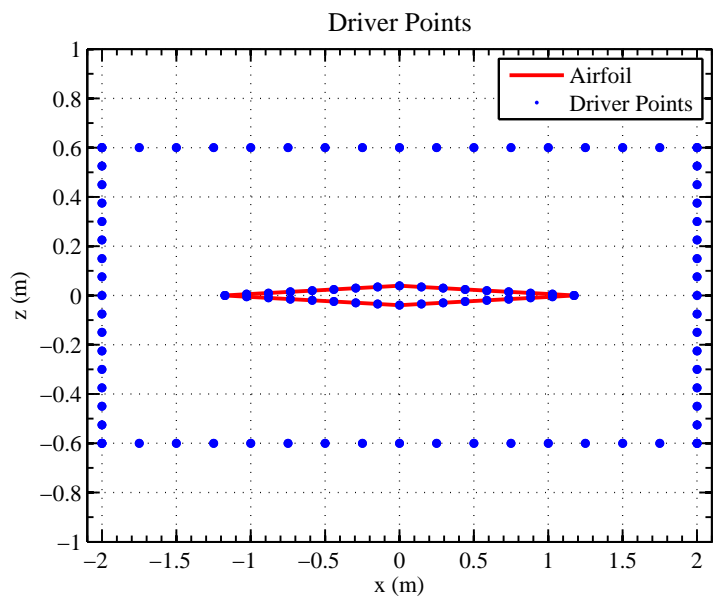

(b) Driving points

Figure 7. Grid deformation using 96 points and fixed outer boundaries

This simple example involves only rigid body displacements and thus does not require a FE model. Two different strategies are considered to deformed the mesh: (1) a moving mesh with fixed outer boundaries and (2) a fully moving mesh. The first mesh deformation strategy enforces fixed outer boundaries, which may be a requirement due to the imposition of boundary conditions in the CFD solver. The driving points are uniformly spaced on the airfoil surface and around the outer boundary of the fluid domain, as shown in Fig. 7(b) in the case of 96 points, 17 on each boundary. The deformed mesh is depicted in Fig. 7(a) for 
a large deformation corresponding to a pitch angle of $\alpha=10^{\circ}$. This deformation is not representative of the small one that occurs in the calculation of the transient response of the aeroelastic system. However it shows the smoothness of the mesh deformation based on RBF. It can also be noted that wiggles appear at the top and bottom boundaries and at the airfoil surface. Despite their relatively small amplitudes, it is necessary to quantify the error introduced by the RBF mesh deformation. Since the airfoil undergoes rigid body motions, the analytical form of the deformation is known and is compared to the one given by the RBF for different RF. For each mode, the airfoil is deformed and both deformations are compared. The results are presented in Figs 8(a) and 8(b) for two different sets of driving points. In both cases, the driving points are located at the boundaries of the CFD mesh as depicted in Fig. 7(b). In Fig. 8(a), 96 points are used to drive the mesh. The maximum error varies from $1 \%$ of the maximum deflection up to $3.4 \%$ depending on the RF chosen. Volume spline, Euclid's hat and Wendland's $C^{2} \mathrm{RF}$ give the best approximation. This result shows that those RF are the one able to approximate a linear interpolation more accurately. In Fig. 8(b), the case of 426 driving points are considered. The error is decreased by more than an order of magnitude to less than $0.05 \%$ for most of the RF's. It shows that in the case of constrained outer boundary, the number of driving points need to be carefully chosen to preserve the shape of the moving airfoil.

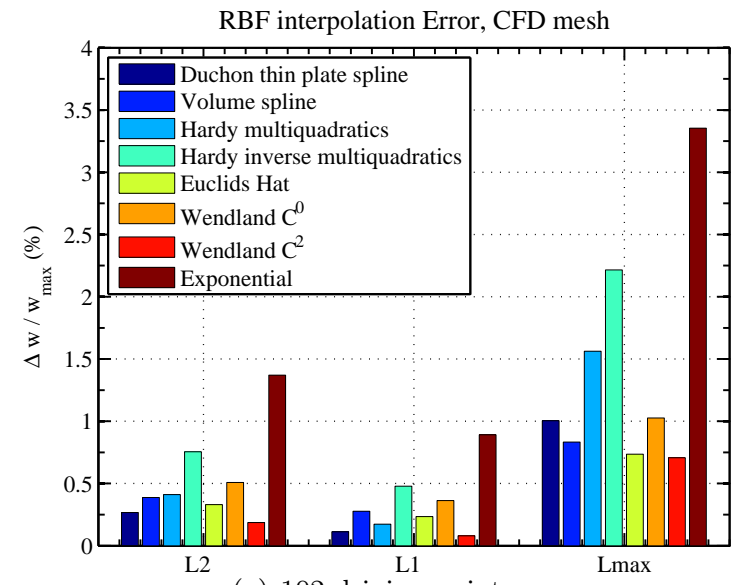

(a) 102 driving points

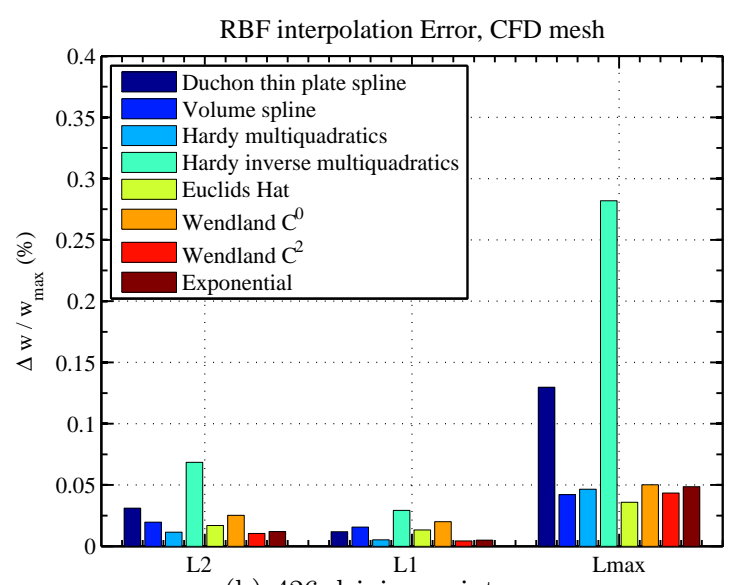

(b) 426 driving points

Figure 8. Effect of the choice of the radial basis function

The second mesh strategy considers a fully moving mesh approach. In the case of the typical section, the number of driving points is not important as long as there are sufficient points to capture the rigid body motion. In theory, 3 non-collinear points are sufficient, in practice a set of 10 points is considered to ensure a better conditioning of the RBF system, given by Eq. (18): the leading and trailing edges, both midchord points on the top and bottom surface of the airfoil and 6 additional points far from the airfoil. The deformations associated with both rigid body motions of the airfoil, plunge and pitch, are computed exactly. As mentioned in Section II.B, a first order polynomial in the form of the RBF interpolation ensures that it is the case.

Based on this preliminary results on mesh deformation, Volume spline is the chosen RF and the mesh is a fully moving mesh. Next two representative cases are considered to verify the new SI method.

The aeroelastic simulations are performed at constant altitude of $12 \mathrm{kms}$ and different Mach numbers. The parameters related to the time stepping and the computational cost for an aeroelastic transient simulation are given in Table 5. The main contributor to the computational cost associated with the aeroelastic simulations is the CFD calculation. Each aeroelastic simulation requires 2500 initial iterations to converge the steady state solution of the $\mathrm{N}-\mathrm{S}$ equations. For each time step, several subiterations of the CFD solver are required to march the fluid in time as explained previously. At each time step, the new node locations are written in the mesh file and the CFD code is restarted. It generates significant amount of additional processing time which is not needed in a fully integrated aeroelastic solver.

For each Mach number, the frequencies and damping are computed using each SI method presented in Section III. The results corresponding to Euler aerodynamics are presented first in Figs. 9. Frequencies and damping coefficients as functions of the Mach number are shown in Figs. 9(a) and 9(b) respectively. The 


\begin{tabular}{cc}
\hline \hline Number of time step & 1000 \\
Time step & $0.125 \mathrm{~ms}$ \\
Number of subiterations & 30 \\
per time step & $45 \mathrm{k}$ \\
Number of cells & 2 \\
\hline Number of processors & Intel(R) Xeon $(\mathrm{R})$ \\
Processor & CPU X5650, 2.67GHz \\
Computational time & $9 \mathrm{hrs}$ \\
\hline \hline
\end{tabular}

Table 5. Parameters and computational cost of 2D calculations using Navier-Stokes aerodynamics

symbols correspond to the Mach numbers at which a transient simulation is performed. Each type of symbol and color correspond to a different mode. The different types of line correspond to different SI methods. The thicker line corresponds to the results obtained using $3^{\text {rd }}$ order PT combined with ARMA. Therefore to each mode correspond 1 color, 1 symbol and 4 different line types, 3 for CFD results and 1 for PT. The symbols correspond to the Mach numbers at which the aeroelastic simulations are computed using Euler aerodynamics. The three SI methods predict identical frequencies and damping coefficients, therefore the three corresponding lines cannot be distinguished. The predicted flutter Mach number is 12.1 which is in good agreement with the one obtained using piston theory. This results is expected as piston theory is a good approximate model for thin bodies oscillating in hypersonic flows.

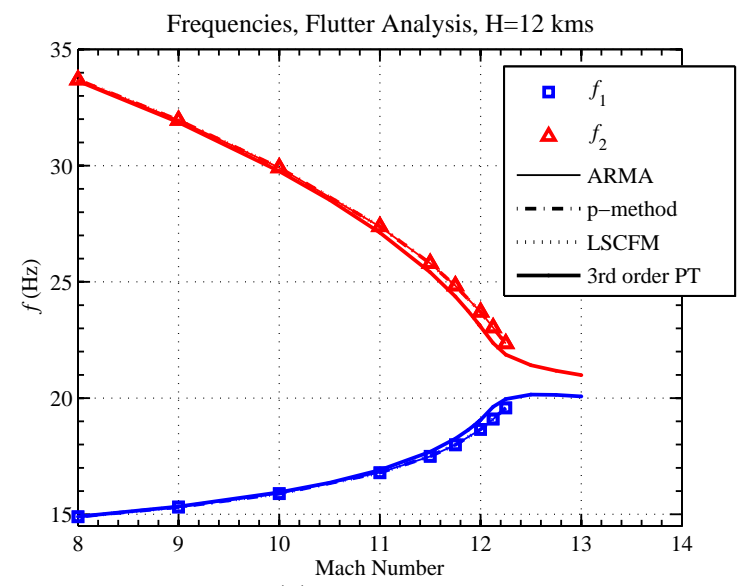

(a) Frequencies

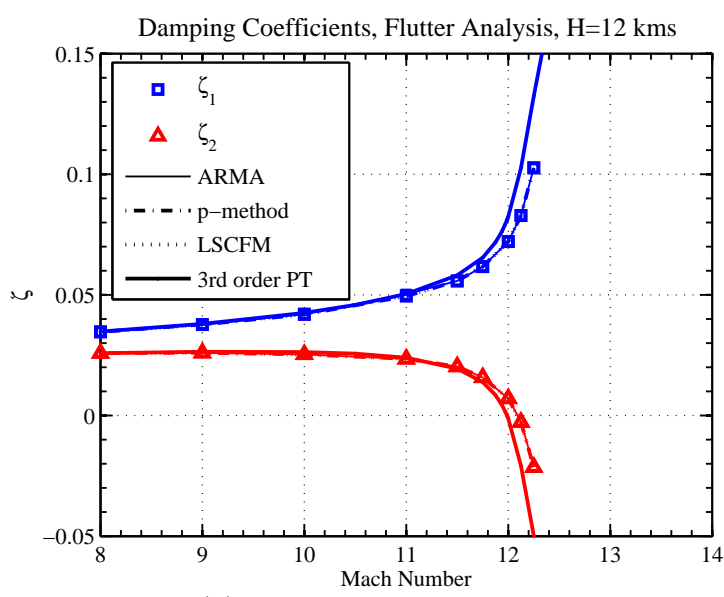

(b) Damping Coefficients

Figure 9. Aeroelastic stability analysis using Euler aerodynamics for the typical section, $\mathrm{H}=12 \mathrm{kms}$

Next, viscosity is introduced and the results are presented in Fig. 10. The crosses refer to the results computed using CFL3D in Ref. 21. The symbols correspond to the Mach numbers considered. The different lines correspond to the different SI methods which are almost identical before flutter occurs at Mach $M_{\infty} \simeq$ 11.3. The damping coefficients are more sensitive to the SI method. The flutter Mach number predicted by each method and including the different moving mesh strategies are given in detail in Table 6. Adding more driving points ensure that the motion of the airfoil are captured more accurately and the flutter prediction converges toward the fully moving mesh approach. The ARMA approach is most sensitive whereas the other two methods are always in agreement with each other. For LSCFM and p-method, the flutter Mach number is predicted within less than $1 \%$ depending on the mesh deformation strategy.

This preliminary study illustrates the use of radial basis functions in a typical aeroelastic calculation and verifies the usage of the SI method. Compared to the results obtained with CFL3D, the flutter onset occurs at a lower Mach number. The difference is approximately $6 \%$ and is explained next by considering additional 


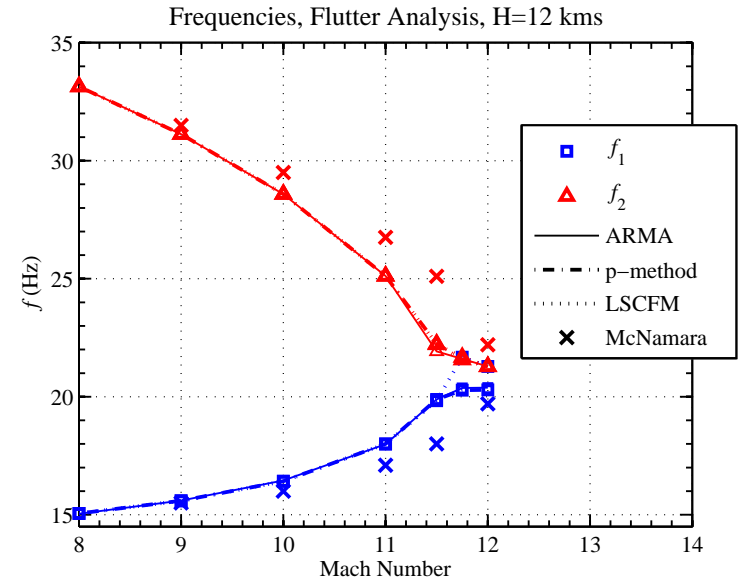

(a) Frequencies

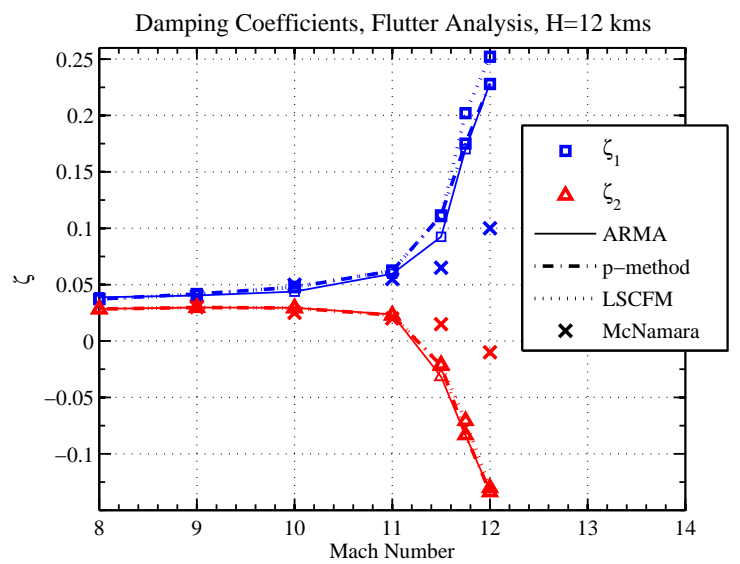

(b) Damping Coefficients

Figure 10. Aeroelastic stability analysis using $\mathrm{N}-\mathrm{S}$ aerodynamics for the typical section, $\mathrm{H}=12 \mathrm{kms}$

\begin{tabular}{c|cc|cc}
\hline \hline & Number of driving points & ARMA & LSQCF & p-method \\
\hline All moving & 10 & 11.21 & 11.26 & 11.26 \\
\hline \multirow{2}{*}{ Fixed outer boundary } & 96 & 11.47 & 11.32 & 11.32 \\
& 420 & 11.42 & 11.27 & 11.27 \\
& CFL3D & \multicolumn{2}{|c}{11.9} & $\mathrm{~N} / \mathrm{A}$ \\
\hline \hline
\end{tabular}

Table 6. Flutter Mach number for the typical section using Navier-Stokes aerodynamics, $H=12 \mathrm{kms}$

cases. Note that in all cases presented next, the three SI methods predict the same flutter Mach number.

Considering different turbulence models provides more details to explain the difference between the calculations performed with CFL3D and the current framework. In both CFL3D and CFD++, the SpalartAllmaras turbulence model ${ }^{31}$ is available and is used for the flutter calculations. In CFL3D, the additional equation does not require any user input and the intensity of the freestream turbulence is set by the ratio $\left(\frac{\mu_{T}}{\mu_{L}}\right)_{\infty}=0.009$ as boundary and initial condition of the turbulence equation. However, in $\mathrm{CFD}++$, this parameter is a user input. The effect of turbulence on flutter boundary prediction is investigated for various flight conditions. Euler and laminar flow are compared to turbulent flow for low and high values of free stream turbulence. The one equation Goldberg turbulence model is also considered. Details of the results are given in Table. 7. Note that adding turbulence decreases the flutter boundary by up to 7\%. An abrupt change is observed when comparing the results for laminar flow, which is close to Euler, to the ones for turbulent flow. The flutter Mach number predicted in the case of SA model with $\left(\frac{\mu_{T}}{\mu_{L}}\right)_{\infty}=1$ is close to the one with $\left(\frac{\mu_{T}}{\mu_{L}}\right)_{\infty}=10$. However the case of Goldberg turbulence model and $\left(\frac{\mu_{T}}{\mu_{L}}\right)_{\infty}=1$ compares well with laminar flow. It illustrates the variability associated with turbulence modeling. Note that the decrease of flutter boundary with increasing turbulence can be explained by the following: The Mach number contours reveal a thicker boundary layer for the more turbulent cases. It yields higher static pressures on the airfoil due to the thicker effective shape and explain the decrease in flutter onset. The effect of gas modeling is negligible for the flight condition considered.

Additional results at different altitudes are presented in Figs. 11. The trends presented are confirmed at higher and lower altitudes. In Fig. 11(a), the effect of turbulence is considered. Both lines corresponding to piston theory and laminar flow coincide and predict the higher flutter boundary at all altitudes. Turbulence is added considering the SA model and the cases of $\left(\frac{\mu_{T}}{\mu_{L}}\right)_{\infty}=0.009$ (low T.) and $\left(\frac{\mu_{T}}{\mu_{L}}\right)_{\infty}=10$ (high T.). The lowest flutter onset is predicted for high turbulence flow. For low turbulence, the flutter onset is close to the case of high turbulence for all altitudes but the higher one. In Fig. 11(b), the effect of gas modeling 


\begin{tabular}{|c|c|c|c|c|c|c|}
\hline Gas Model & Fluid Model & Turbulence Model & $\left(\frac{\mu_{T}}{\mu_{L}}\right)_{\infty}$ & ARMA & LSCFM & $\mathrm{p}$-method \\
\hline \multirow{7}{*}{$\mathrm{PG}$} & $\mathrm{EU}$ & - & - & 12.09 & 12.09 & 12.09 \\
\hline & \multirow{6}{*}{ NS } & Laminar & - & 11.82 & 11.83 & 11.83 \\
\hline & & $\mathrm{SA}$ & 0.009 & 11.63 & 11.63 & 11.63 \\
\hline & & $\mathrm{SA}$ & 3 & 11.47 & 11.50 & 11.43 \\
\hline & & $\mathrm{SA}$ & 10 & 11.21 & 11.26 & 11.26 \\
\hline & & \multirow{2}{*}{ Goldberg } & 1 & 11.80 & 11.81 & 11.81 \\
\hline & & & 10 & 11.15 & 11.15 & 11.15 \\
\hline IG & NS & SA & 3 & 11.53 & 11.52 & 11.52 \\
\hline $\mathrm{RG}$ & NS & $\mathrm{SA}$ & 3 & 11.50 & 11.46 & 11.50 \\
\hline
\end{tabular}

Table 7. Flutter Mach number for the typical section using different turbulence and gas models, $H=12 \mathrm{kms}$

is considered. Turbulence is added considering the SA model and the case of $\left(\frac{\mu_{T}}{\mu_{L}}\right)_{\infty}=3$. The three lines corresponding to PG, IG, and RG model coincide.

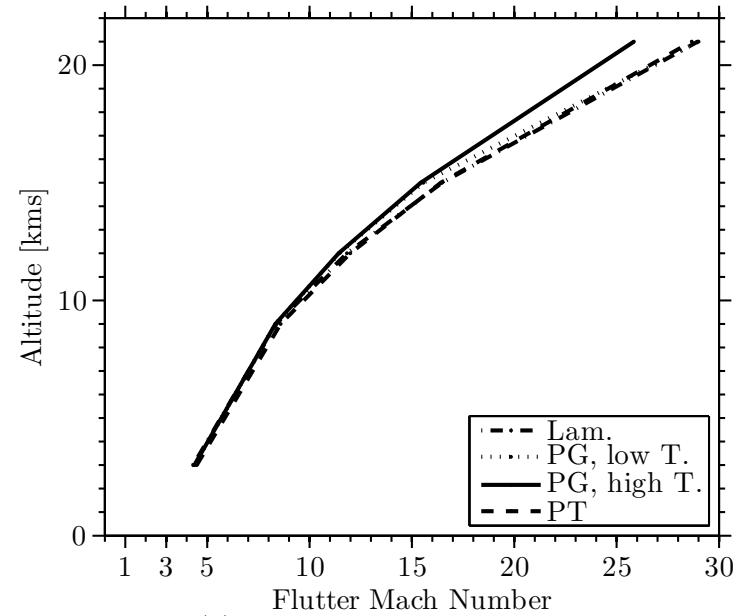

(a) Effect of turbulence flow

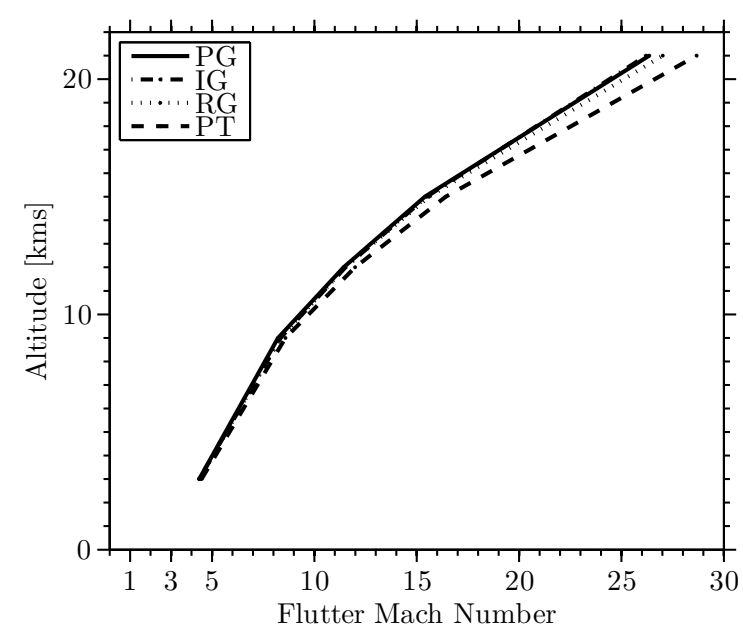

(b) Effect of gas model

Figure 11. Aeroelastic stability analysis for the typical section for different turbulent flow, $\mathrm{H}=12 \mathrm{kms}$

In conclusion, turbulence is the main contributor to the variability in flutter Mach number. Real gas effects do not affect the flutter boundary significantly. Next the three dimensional low aspect ratio wing is considered.

\section{IV.B. Three Dimensional Low Aspect Ratio Wing}

The structure of the wing is represented by five natural modes depicted in Fig. 3. The 1327 FEM nodes are uniformity distributed at the surface of the wing as illustrated in Fig. 12(a).

In the $3 \mathrm{D}$ case, the analytical form of the mode shape is not available since they are obtained by a FEM calculation. Wiggles are likely to appear when using the RBF mesh deformation based on the FEM points, therefore it is important to quantify their amplitude. To assess this issue, the deformed wing configuration using RBF is compared with the one obtained with a piecewise linear interpolation between the FEM nodes for the second mode shape, $\phi_{2}$. In Table. 8, two cases are considered: (1) the driving points are the FEM nodes and (2) the driving points are the CFD nodes at the wing interface. First, the driving points at the surface of the wing are the FEM nodes. The maximum difference between the deformation obtained with $\mathrm{RBF}$ and the one based on piecewise linear interpolation is of $0.9 \%$ and $1.4 \%$ for a moving and fixed outer 


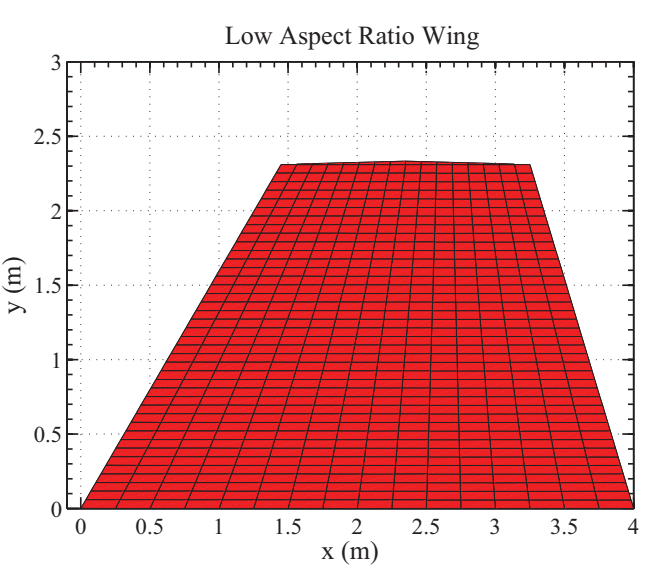

(a) Finite element mesh

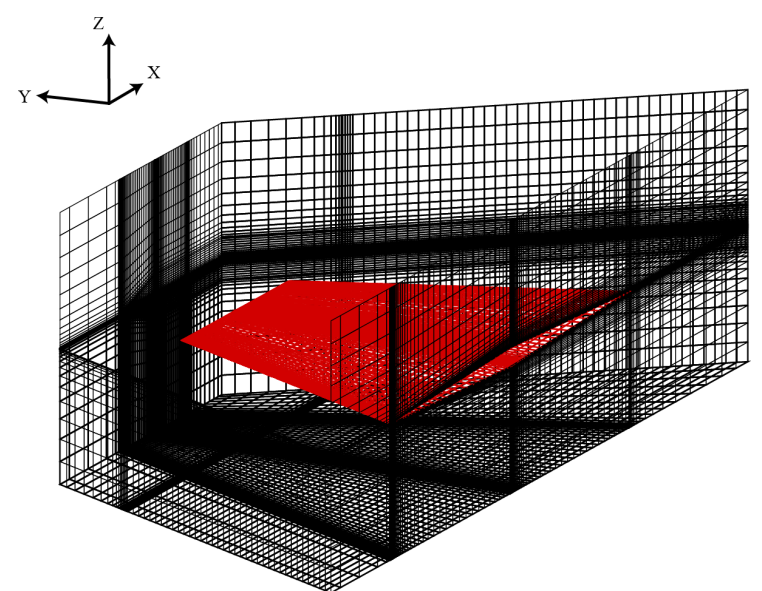

(b) Grid 3

Figure 12. Meshes for the low aspect ratio wing

boundary, respectively. Adding driving points to prescribe a static outer boundary significantly affects the shape of the deformed wing. Similarly the mode shape calculated at the centroid of the faces which lie on the wing is affected and the difference is of the same order of magnitude. For the second case, the driving points at the surface of the wing are the fluid mesh points. As expected, the difference between the RBF and the reference shape is reduced to 0. Similarly for the centroid, there is good agreement between RBF and piecewise linear interpolation. This example demonstrates some of the issues associated with RBF mesh deformation. For the results presented in this report, the CFD nodes are the driving points. Volume spline is the RF of choice and the mesh is fully moving.

\begin{tabular}{ccccc|ccc}
\hline \hline & & \multicolumn{3}{|c|}{ Fixed outer boundaries } & \multicolumn{3}{|c}{ Moving outer boundaries } \\
& & $L_{2}(\%)$ & $L_{1}(\%)$ & $L_{\infty}(\%)$ & $L_{2}(\%)$ & $L_{1}(\%)$ & $L_{\infty}(\%)$ \\
\hline \multirow{2}{*}{ FEM based } & Fluid Mesh & 0.214 & 0.09 & 1.4 & 0.114 & 0.053 & 0.887 \\
& Face centroid & 0.21 & 0.10 & 1.26 & 0.109 & 0.060 & 0.768 \\
\hline \multirow{2}{*}{ CFD based } & Fluid Mesh & 0.001 & 0.001 & 0.018 & 0.001 & 0.001 & 0.018 \\
& Face centroid & 0.039 & 0.024 & 0.215 & 0.039 & 0.024 & 0.215 \\
\hline \hline
\end{tabular}

Table 8. Error due to the RBF interpolation

The computational cost and the parameters associated with the time stepping of the aeroelastic simulations are presented in Table. 9. The time step is set to $\Delta t=1.25 \times 10^{-4} \mathrm{~s}$ which corresponds to approximately 100 and 500 time steps per period for the highest and lowest natural frequency respectively. The computational cost associated with the current framework is higher than the one associated with CFL3D for the reasons similar to those detailed in Section IV.A. Depending on the complexity of the gas model ( turbulence, chemistry ) the computational time varies from 110 hours for Euler and laminar flow to 190 when chemistry is included.

The flutter analysis results are presented in Table. 10 for an altitude of $12 \mathrm{kms}$. The LSCFM and pmethod show good agreement. The results for the low aspect ratio wing showed that ARMA has difficulties identifying frequencies and damping of the aeroelastic deformation. In the case of Euler aerodynamics, there is a good agreement with CFL3D and the present results. The predicted flutter Mach number is 13.7. In the case of Navier-Stokes aerodynamics, the flutter difference between CFL3D and the current simulation is up to $7 \%$. The trends observed in the $2 \mathrm{D}$ case are confirmed: adding turbulence decreases the flutter boundary, and real gas effect have a limited effect on the flutter boundary. The results of the flutter analysis are detailed in Figs. 13 and 14.

In Fig. 13, different turbulence modeling assumptions are considered. Figure 13(a) depicts the frequencies of the aeroelastic system as function of flutter Mach number. The different types of lines correspond to 


\begin{tabular}{cc}
\hline \hline Number of time step & 1000 \\
Time step & $0.125 \mathrm{~ms}$ \\
Number of subiterations per time step & 50 \\
Number of cells & $1 \mathrm{M}$ \\
\hline Number of processors & 6 \\
Processor & Opteron $240-254$ \\
Computational time & $110-190 \mathrm{hrs}$ \\
\hline \hline
\end{tabular}

Table 9. Simulations parameters for $3 \mathrm{D}$ calculations

\begin{tabular}{|c|c|c|c|c|c|c|}
\hline Gas Model & Fluid Model & Turbulence Model & $\left(\frac{\mu_{T}}{\mu_{L}}\right)_{\infty}$ & ARMA & LSCFM & p-method \\
\hline \multirow{4}{*}{$P G$} & $\mathrm{EU}$ & - & - & 13.82 & 13.69 & 13.73 \\
\hline & \multirow{3}{*}{ NS } & Laminar & - & 13.5 & 13.32 & 13.30 \\
\hline & & SA & 0.009 & 12.8 & 12.76 & 12.77 \\
\hline & & SA & 1 & 12.46 & 13.00 & 12.78 \\
\hline IG & NS & SA & 3 & 13.03 & 13.03 & 13.02 \\
\hline RG & NS & SA & 3 & 12.77 & 13.01 & 12.91 \\
\hline \multirow{2}{*}{ CFL3D $^{21}$} & $\mathrm{EU}$ & - & - & \multirow{2}{*}{\multicolumn{2}{|c|}{$\begin{array}{c}13.7 \\
13.65\end{array}$}} & $\mathrm{~N} / \mathrm{A}$ \\
\hline & NS & SA & 0.009 & & & $\mathrm{~N} / \mathrm{A}$ \\
\hline \multicolumn{2}{|c|}{$3^{r d}$ order PT } & & & \multicolumn{3}{|c|}{13.4} \\
\hline
\end{tabular}

Table 10. Flutter Mach number for the typical section using different turbulence and gas models, $H=12 \mathrm{kms}$

different turbulence assumptions. In Fig. 13(b), only the damping coefficients corresponding to the first two modes are shown for sake of clarity. The frequencies of the aeroelastic system are not sensitive to the model considered compared to the damping coefficients. Note that the flutter Mach number predicted using CFL3D and Navier-Stokes is smaller than the one obtained with Euler. It confirms the trend that adding turbulence increases the apparent shape due to the presence of the boundary layer and eventually results in an increased local pressure on the wing and a decrease in flutter Mach number.

The results of the flutter analysis are detailed in Fig. 14 for different gas models. Figure 14(a) depicts the frequencies of the aeroelastic system as function of flutter Mach number. The different types of lines correspond to different turbulence assumptions. In Fig. 14(b), the damping coefficients are affected by the gas model. Going from perfect gas to ideal gas affects the flutter boundary by about $3 \%$. The difference between IG and RG is negligible.

When considering these results, it is important to note that the altitude ( $H=12 \mathrm{kms})$ at which the aeroelastic studies are conducted is not representative of hypersonic flight. More realistic altitudes of 20-30 kms produce very high Mach numbers, and therefore the altitude is artificially reduced in order to reduce these to practical values. However, as pointed out in Ref. 22, incorporation of aerodynamic heating leads to a reduction of the flutter Mach number, and thus aerothermoelastic studies can be conducted at more reasonable altitudes.

\section{Conclusions}

A framework for hypersonic aeroelastic calculation using CFD is presented. A detailed description of each component is given. The RBF approach is a robust and efficient mesh deformation method. However, it does not preserve the geometrical shape exactly throughout the deformation if the number of points is not sufficient. This issue needs to be assessed before running aeroelastic simulations. A system identification method for determining the flutter speed has been developed. It is both robust and performs well. Several 
Frequencies, Flutter Analysis, $\mathrm{H}=12 \mathrm{kms}$

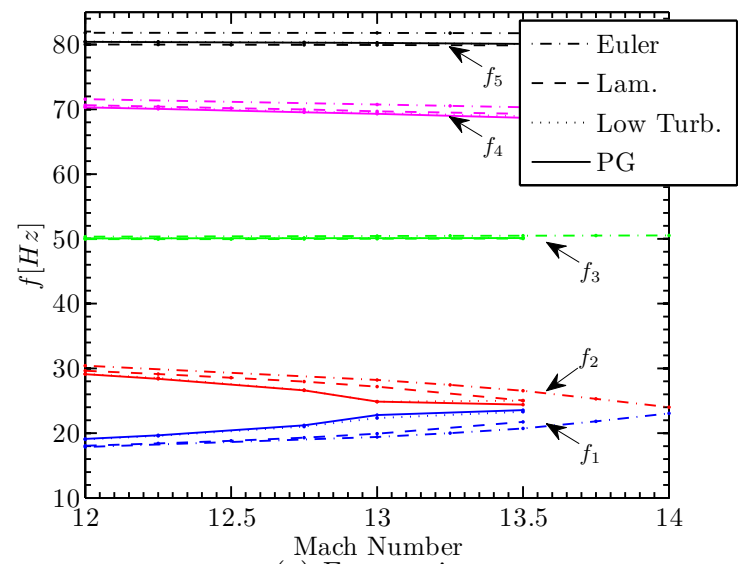

(a) Frequencies

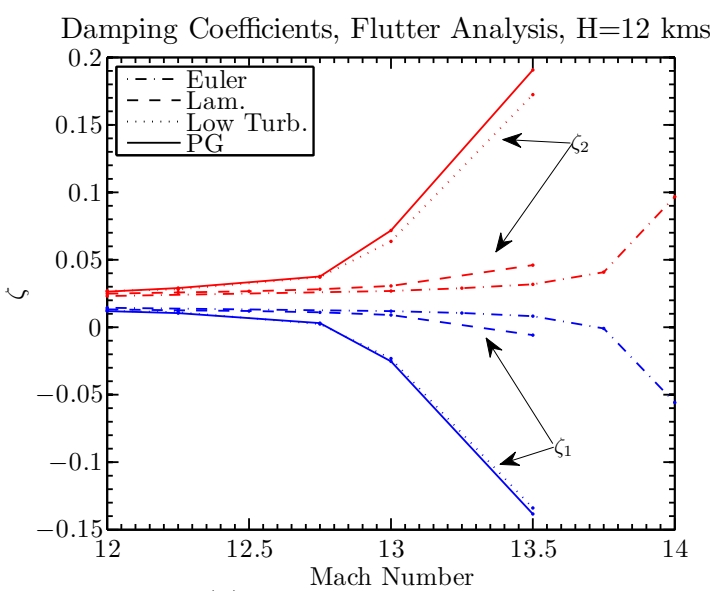

(b) Damping Coefficients

Figure 13. Aeroelastic stability analysis for the low aspect ratio wing for different turbulent flow , H $=12$

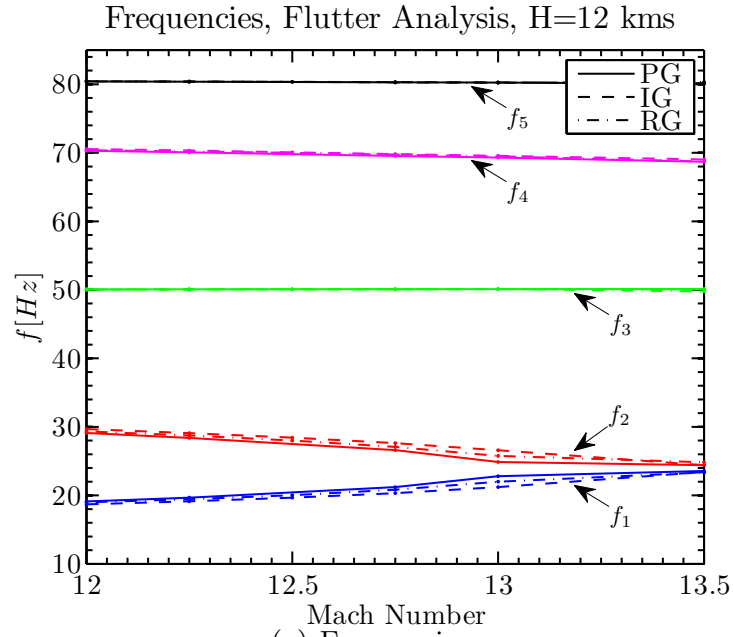

(a) Frequencies

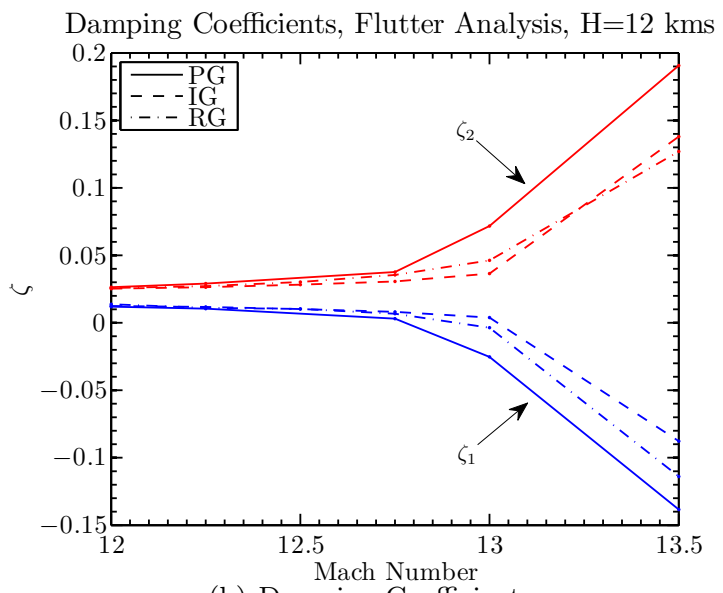

(b) Damping Coefficients

Figure 14. Aeroelastic stability analysis for the low aspect ratio wing for different gas model, $\mathrm{H}=12 \mathrm{kms}$

observations and conclusions can be pointed out:

1. Introducing viscosity reduces the flutter margin: a thicker boundary layer results in an increase in aerodynamic loading.

2. Turbulence modeling introduces variability in the flutter Mach number predictions and prevents its exact calculation.

3. Real gas effects have only a limited effect on flutter Mach number at the flight conditions considered.

\section{Acknowledgements}

This research is funded under NASA grant NNX08AB32A with Donald Soloway and Jorge Bardina as technical monitors. The authors would like to acknowledge the help of Prof. Jack J. McNamara from Ohio State University, and Dr Beerinder Singh from Metacomp Technologies. 


\section{References}

${ }^{1}$ Bertin, J. J., Hypersonic Aerothermodynamics, AIAA Education Series, 1938.

${ }^{2}$ Anderson, J. D., Modern Compressible Flow with Historical Perspective, Third Edition Mc Graw Hill Editions, 2004.

${ }^{3}$ Dugundji, J. and Calligeros, J. M., "Similarity Laws for Aerothermoelastic Testing," Journal of Aerospace Sciences, Vol. 29, No. 8, Aug. 1962, pp. 935-950.

${ }^{4}$ Roy, C. J. and Blottner, F. G., "Review and assessment of turbulence models for hypersonic flows," Progress in Aerospace Sciences, Vol. 42, No. 7-8, 2006, pp. 469-530.

${ }^{5}$ McNamara, J. J. and Friedmann, P. P., "Aeroelastic and Aerothermoelastic Analysis in Hypersonic Flow: Past, Present, and Future," Vol. 49, No. 6, June 2011, pp. 1089-1122.

${ }^{6}$ Farhat, C., Lesoinne, M., and Le Tallec, P., "Load and motion transfer algorithms for fluid/structure interaction problems with non-matching discrete interfaces: momentum and energy conservation, optimal discretization and application to aeroelasticity," Computer methods in applied mechanics and engineering, Vol. 157, No. 1-2, 1998, pp. 95-114.

${ }^{7}$ Bendiksen, O. O., "A new approach to computational aeroelasticity," AIAA/ASME/ASCE/AHS/ASC Structures, Structural Dynamics, and Materials Conference, 32 nd, Baltimore, MD, Technical Papers. Pt. 3, Vol. 8, 1991.

${ }^{8}$ Bendiksen, O. O., "Modern developments in computational aeroelasticity," Proceedings of the Institution of Mechanical Engineers, Part G: Journal of Aerospace Engineering, Vol. 218, No. 3, 2004, pp. 157-177.

${ }^{9}$ Bendiksen, O. O. and Seber, G., "Fluid-structure interactions with both structural and fluid nonlinearities," Journal of Sound and Vibration, Vol. 315, No. 3, 2008, pp. 664-684.

${ }^{10}$ Farhat, C., van der Zee, K. G., and Geuzaine, P., "Provably second-order time-accurate loosely-coupled solution algorithms for transient nonlinear computational aeroelasticity," Computer Methods in Applied Mechanics and Engineering, Vol. 195, No. 17-18, 2006, pp. 1973 - 2001, Fluid-Structure Interaction.

${ }^{11}$ Rendall, T. and Allen, C., "Unified fluid-structure interpolation and mesh motion using radial basis functions," International Journal for Numerical Methods in Engineering, Vol. 74, No. 10, 2008, pp. 1519-1559.

${ }^{12}$ Farhat, C. and Lesoinne, M., "Two efficient staggered algorithms for the serial and parallel solution of three-dimensional nonlinear transient aeroelastic problems," Computer Methods in Applied Mechanics and Engineering, Vol. 182, No. 3-4, 2000, pp. $499-515$.

${ }^{13}$ van Zuijlen, A., de Boer, A., and Bijl, H., "Higher-order time integration through smooth mesh deformation for 3D fluid-structure interaction simulations," Journal of Computational Physics, Vol. 224, No. 1, 2007, pp. $414-430$.

${ }^{14}$ Morris, A., Allen, C., and Rendall, T., "CFD-based optimization of aerofoils using radial basis functions for domain element parameterization and mesh deformation," International Journal for Numerical Methods in Fluids, Vol. 58, No. 8, 2008, pp. 827-860.

${ }^{15}$ Van Zuijlen, A., De Boer, A., and Bijl, H., "Higher-order time integration through smooth mesh deformation for 3D fluid-structure interaction simulations," Journal of Computational Physics, Vol. 224, No. 1, 2007, pp. 414-430.

${ }^{16}$ Rendall, T. and Allen, C., "Efficient mesh motion using radial basis functions with data reduction algorithms," Journal of Computational Physics, Vol. 228, No. 17, 2009, pp. 6231-6249.

${ }^{17}$ Jakobsson, S. and Amoignon, O., "Mesh deformation using radial basis functions for gradient-based aerodynamic shape optimization," Computers and Fluids, Vol. 36, No. 6, 2007, pp. 1119 - 1136.

${ }^{18}$ de Boer, A., van Zuijlen, A., and Bijl, H., "Review of coupling methods for non-matching meshes," Computer Methods in Applied Mechanics and Engineering, Vol. 196, No. 8, 2007, pp. 1515 - 1525.

${ }^{19}$ de Boer, A., van Zuijlen, A., and Bijl, H., "Comparison of conservative and consistent approaches for the coupling of non-matching meshes," Computer Methods in Applied Mechanics and Engineering, Vol. 197, No. 49-50, 2008, pp. 4284 - 4297.

${ }^{20}$ Buhmann, M. D., Radial Basis Functions: Theory And Implementations, Cambridge: Cambridge University Press, 2003.

${ }^{21}$ McNamara, J. J. and Friedmann, P. P., "Flutter Boundary Identification for Time-Domain Computational Aeroelasticity," AIAA Journal, Vol. 45, No. 7, July 2007, pp. 1546-1555.

${ }^{22}$ McNamara, J. J., Friedmann, P. P., Powell, K., Thuruthimattam, B., and Bartels, R., "Aeroelastic and Aerothermoelastic Behavior in Hypersonic Flow," AIAA Journal, Vol. 46, No. 10, Oct. 2008, pp. 2591-2610.

${ }^{23}$ Krist, S., Biedron, R., and Rumsey, C., CFL3D user's manual (version 5.0), NASA/TM-1998-208444, September 1998.

${ }^{24}$ Bartels, R., Rumsey, C., and Biedron, R., "CFL3D Version 6.4, General usage and aeroelastic analysis," Tech. rep., NASA TM-2006-214301, 2006.

${ }^{25}$ Higham, N. J., "The Scaling and Squaring Method for the Matrix Exponential," SIAM Journal on Matrix Analysis and Applications, Vol. 26, No. 4, 2005, pp. 1179-1193.

${ }^{26}$ Park, C., Nonequilibrium hypersonic aerothermodynamics, Wiley-Interscience New York etc., 1990.

${ }^{27}$ Pak, C. G. and Friedmann, P. P., "New time-domain technique for flutter boundary identification," AIAA Dynamics Specialists Conference, Vol. 201-214, AIAA, Dallas, TX, April 1992, pp. 201-214.

${ }^{28}$ Hassig, H. J., "An approximate true damping solution of the flutter equation by determinant iteration." Journal of Aircraft, Vol. 8, No. 11, Nov. 1971, pp. 885-889.

${ }^{29}$ Rodden, W. P., Chapter 7, Theoretical and Computational Aeroelasticiy, Crest Publishing, 2011.

${ }^{30}$ Crassidis, J. L. and Junkins, J. L., Optimal Estimation of Dynamic Systems, Chapman \& Hall/CRC Applied Mathematics and Nonlinear Science Series, 2004.

${ }^{31}$ Spalart, P. R. and Allmaras, S. R., "A One-Equatlon Turbulence Model for Aerodynamic Flows," Proceedings of the 30th Aerospace Sciences Meeting and Exhibit, Reno, NV, Jan. 1992. 